Preprints of the

Max Planck Institute for

Research on Collective Goods

Bonn 2005/23

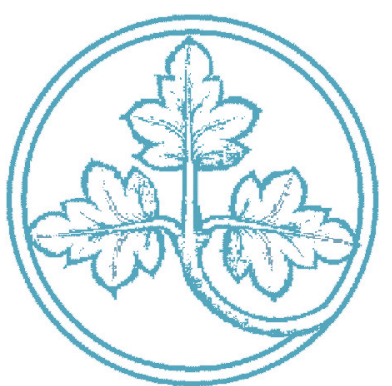

A Contribution to the Theory of Optimal Utilitarian Income Taxation

Martin F. Hellwig

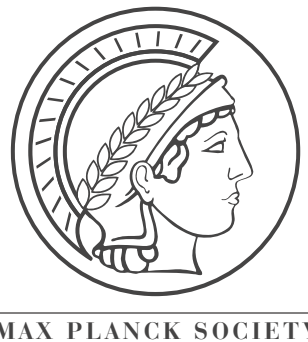




\title{
A Contribution to the Theory of Optimal Utilitarian Income Taxation
}

\author{
Martin F. Hellwig
}

November 2005 


\title{
A Contribution to the Theory of Optimal Utilitarian Income Taxation*
}

\author{
Martin F. Hellwig \\ Max Planck Institute for Research on Collective Goods \\ Kurt-Schumacher-Str. 10, D - 53113 Bonn, Germany \\ hellwig@coll.mpg.de
}

October 31, 2005

\begin{abstract}
The paper provides a new proof of the positivity of the optimal marginal income tax, in a more general model, under weaker assumptions. The analysis focusses on the (weakly) relaxed problem in which upward incentive constraints are replaced by a monotonicity condition on consumption. Without upward incentive constraints, nonnegativity of the optimal marginal income tax is straightforward; strict positivity follows from an assumption on the desirability of redistributing leisure. The resulting allocation is incentive compatible, and is optimal for the original income tax problem. The argument is the same for distributions with finitely many types and for a continuous type distribution.

Key Words: Optimal Income Taxation, Utilitarian Welfare Maximization, Redistribution

JEL Classificaton: D63, H21
\end{abstract}

\section{Introduction}

The central result of the theory of optimal utilitarian income taxation states that, under asymmetric information, when individual productivity (earning ability) is a hidden characteristic, the optimal marginal income tax is strictly positive at all income levels other than the very top and, in some specifications, the very bottom of the income distribution. The first version of this

${ }^{*}$ I am very grateful to Felix Bierbrauer, Christoph Engel, Thomas Gaube, and Peter Norman for very helpful discussions and comments on earlier drafts. The usual disclaimer applies. 
result was proved by Mirrlees $(1971,1976)$ for a model with a continuum of types; his result has subsequently been generalized by Seade $(1977,1982)$, Ebert (1992), and Brunner (1993, 1995). For models with finitely many types, different versions of the result have been provided by Guesnerie and Seade (1982), Stiglitz (1982), Roëll (1985), and Weymark (1986).

In this paper, I develop a new approach to proving this result. The new approach provides for a sharper formulation of the theorem, in a more general model, under weaker assumptions. More importantly, it provides for a transparent account of the underlying logic, relating the mathematics to the economics and making clear where exactly and how the key assumptions enter the analysis. In contrast to the literature, the overall proof strategy is the same for models with finitely many types and with a continuum of types. Moreover, for models with a continuum of types, there is no need to have different arguments for different cases. In contrast, Seade's (1982) analysis, on which most subsequent work has built, ${ }^{1}$ involves separate treatments depending on whether consumption and leisure are (Edgeworth) substitutes or complements; this makes it hard to see the common underlying structure.

The new approach proceeds indirectly. Following Matthews and Moore (1987), I study a modification of the optimal income tax problem, which is obtained if upward incentive constraints are replaced by a monotonicity condition on consumption. Under suitable assumptions, I show that the modified problem is equivalent to the optimal income tax problem in the sense that, up to modifications on a null set, any solution to one problem is also a solution to the other and vice versa.

In the modified problem, it is straightforward to show that optimal marginal income taxes are never negative. Negative marginal income taxes, i.e., a subsidization of work at the margin, would induce people to work and to consume more than is efficient. ${ }^{2}$ Such a distortion could only be justified by upward incentive constraints requiring workloads to be fixed with a

\footnotetext{
${ }^{1}$ E.g., Ebert (1992), Brunner (1993, 1995).

${ }^{2}$ This argument assumes that, as in Mirrlees (1971), consumption-leisure choices are made at the intensive margin. Saez (2002) has shown that negative marginal income taxes can be optimal if consumption-leisure choices are made at the extensive margin, so people of type $\mathrm{n}$ choose only whether to work at a job of type $\mathrm{n}$ or not to work; see also Laroque (2005). In this case, there is no question of a person of type $\mathrm{n}$ choosing to work at a job of type n-1 and earning a lower income; marginal income taxes as such are therefore economically unimportant. The optimal tax on income earned on a job of type $n$ is obtained from a simple elasticities consideration involving only people of type n. A model involving choices at both the intensive and the extensive margins would have to allow for multidimensional type heterogeneity; the proper treatment of incentive compatibility in such a model is as yet an open issue.
} 
view to preventing people with lower earning abilities from imitating people with higher earning abilities. When upward incentive constraints are not imposed, such considerations cannot play a role.

The fact that one does not have to worry about negative marginal taxes makes it easier to see under what conditions it is desirable to have strictly positive marginal taxes. This makes it possible to widen the scope, sharpen the conclusions, and weaken the key assumption of the main theorem.

Since Mirrlees (1971), papers on optimal income taxation with a continuum of types have usually assumed that the unknown productivity parameter corresponds to the wage rate that a person earns. In contrast, for models with a finite number of types, Guesnerie and Seade (1982) have introduced a more abstract formulation in which the productivity parameter is simply an argument of the utility function which affects attitudes towards consumption and output provision. The present paper uses the abstract formulation of Guesnerie and Seade (1982) for models with a continuum, as well as a finite number of types. The greater abstractness of the formulation necessitates a more abstract formulation of the underlying assumptions. This contributes to clarifying their respective roles.

The key assumption providing the rationale for utilitarian redistribution is formulated in local terms. Moreover, this assumption is formulated in terms of redistributing leisure rather than consumption. I postulate that, in the absence of incentive considerations, certain allocations cannot be welfare maximizing because, at any one of these allocations, utilitarian welfare can be increased by redistributing leisure from people with a given productivity level to people with slightly higher productivity levels, allowing the former to work less and requiring the latter to work more so that total output is unchanged. This postulate is imposed for all allocations with consumptionleisure choices that are efficient for people with the lower productivity level and either efficient or distorted in the direction of too little consumption and too much leisure for people with the higher productivity levels.

Under this assumption, I show that, if the type set is finite, the optimal marginal income tax is strictly positive for all types below the top. If the type set is an interval and the distribution of types has a continuous and strictly positive density on this interval, the optimal marginal income tax is strictly positive and bounded away from zero on any compact set of types between the bottom and the top of the distribution. In contrast to past treatments, this result does not leave open the possibility that the optimal marginal income tax might be zero at or arbitrarily close to zero near some 
isolated point. ${ }^{3}$

These results can be understood in terms of a local equity-efficiency tradeoff. If the marginal tax rate that is relevant for a given type is zero, then this type's consumption-leisure choice is efficient. Given the information that marginal tax rates are never negative, any higher type's consumptionleisure choice is either efficient or distorted in the direction of too little consumption and too much leisure. The assumption on the desirability of redistribution therefore implies that, if the relevant marginal tax rate for any type is zero, then it is desirable to redistribute output requirements from people with this type to people with slightly higher types. Ceteris paribus, such a redistribution may violate downward incentive compatibility. However, the incentive effects can be neutralized by distorting the lower type's consumption-leisure choice in the direction of too little consumption and too much leisure. The question then is whether the efficiency loss of this shift away from an efficient consumption-leisure pair is outweighed by the postulated gain from the redistribution. By standard arguments, this is always the case because, for a small shift away from efficiency, the efficiency loss is negligible. ${ }^{4}$

In formulating the key assumption on the desirability of redistribution exclusively in terms of leisure, I depart from the traditional utilitarian notion that it is desirable to redistribute consumption because people with higher consumption have a lower marginal utility of consumption. In the theory of income taxation, this notion goes back (at least) to Edgeworth (1897/1925). ${ }^{5}$ Mirrlees (1971, 1976), as well as Guesnerie and Seade (1982) and Weymark (1986), have followed this tradition. They recognized that differences in consumption levels which are due to differences in the hid-

\footnotetext{
${ }^{3}$ Thus, Seade (1982) proceeds by assuming that positivity fails on some interval and obtains a contradiction by comparing the consumption-income pairs that are associated with the two endpoints of the interval. This argument neglects the possibility that the interval in question might be degenerate, i.e., consist of a single point, in which case the comparison in question is moot.

${ }^{4}$ The argument presumes that the two sets of people that are involved are of commensurate sizes. This presumption holds for the specifications studied in this paper. However, it does not hold if the two sets of people that are involved are not of commensurate sizes, in particular, if the lower type has positive mass and any nearby higher type has zero mass. In this case, the aggregate potential gains from redistributing output requirements between people with these types are zero and do not outweigh the efficiency loss from reducing consumption and increasing leisure for the mass of people with the lower type. Thus, in Hellwig (2005 b), I show that, for a type distribution involving mass points, as well as an interval support, the optimal marginal tax for a type with positive mass is zero unless there is bunching.

${ }^{5}$ For a comprehensive account, see Chapter 5 of Musgrave (1959).
} 
den productivity parameters are likely to be correlated with differences in leisure, but, through additional assumptions, they avoided the complications that this might cause. Thus, in Mirrlees (1971), additive separability of utility functions eliminates the possibility that people with higher consumption may be hungrier, even at the margin, because these people also work more.

The focus on the desirability of redistributing consumption was loosened by Seade (1982) and Roëll (1985). For the model of Mirrlees (1971), Seade (1982) showed that the assumption of additive separability of the utility function can be replaced by the assumption that leisure is a noninferior good. For the more abstract utility specification of Guesnerie and Seade (1982), Roëll (1985) showed that the traditional redistribution assumption in terms of consumption can be weakened to the assumption that it is desirable to redistribute consumption or leisure from high-productivity to low-productivity participants. ${ }^{6}$ She also showed that, for the utility specification of Mirrlees (1971), this condition is implied by noninferiority of leisure. ${ }^{7}$

This paper goes one step further and eliminates any reference to the desirability of redistributing consumption. At first sight, this may seem more restrictive than Roëll's formulation: Where she postulates the desirability of redistributing consumption or leisure, I postulate the desirability of redistributing leisure. However, I impose this condition on a smaller set of initial allocations. For the initial allocations on which I impose it, the desirability of redistributing consumption necessarily also implies the desirability of redistributing leisure, i.e., the alternative that Roëll allows for is moot. For the utility specification of Mirrlees (1971), with types corresponding to wage rates, the redistribution assumption which I use is actually weaker than all the assumptions that have been used previously. The weakening is possible because the indirect approach to the optimal income tax problem eliminates many allocations from consideration before one ever gets to questions of redistribution.

For the model with a continuum of types, the paper also provides a technical generalization. Relying on a reformulation of Pontryagin's maximum principle for problems with monotonicity constraints on controls, I can do without requirements such as continuity or piecewise continuous differentiability of optimal allocations and optimal tax schedules, which have hitherto been imposed. Given that optimal allocations and optimal tax schedules are

\footnotetext{
${ }^{6}$ In the formal statement of her theorem, Roëll only claims nonnegativity of the optimal marginal income tax. However, the arguments given by Guesnerie and Seade (1982) for strict positivity apply to her specification as well.

${ }^{7}$ On this point, see also Brunner $(1993,1995)$ and Homburg (2004).
} 
endogenous, the imposition of such conditions by fiat is problematic.

In the following, Section 2.1 formulates the optimal and relaxed income tax problems. Section 2.2 states the assumptions which I impose and relates them to the assumptions imposed in the literature. Section 3 states and explains the main results. Section 4 provides a proof for the case of finitely many types, Section 5 for the case when the type set is an interval and the type distribution has a continuous, strictly positive density. Some supplementary proofs are given in the Appendix.

\section{The Optimal Income Tax Problem}

\subsection{The Problem}

Following Mirrlees (1971, 1976) and Seade (1977, 1982), I study a large economy with one produced good and labour. Each agent in the economy is characterized by a productivity parameter $n$. An agent with productivity parameter $n$ who consumes $c$ units of the produced good and who supplies the labour needed to produce $y$ units of output obtains the payoff $u(c, y, n)$. The leading example in the literature is the specification

$$
u(c, y, n)=U\left(c, \frac{y}{n}\right) .
$$

In this specification, $n$ is labour productivity (the wage rate) and $\frac{y}{n}$ is the number of hours the person needs to work to produce the output $y$ or to obtain the labour income $y$. The analysis here encompasses (2.1), but is not limited to this specification.

The productivity parameter $n$ of any one person is the realization of a nondegenerate random variable $\tilde{n}$ with probability distribution $F$ with compact support $N \subset \Re_{+}$. The distribution $F$ is the same for all agents. By a large-numbers effect, $F$ is also assumed to be the cross-section distribution of the realizations of people's productivity parameters.

In this economy, an allocation is a pair of functions, $(c(\cdot), y(\cdot))$, which indicate how an individual's consumption level $c(n)$ and output provision level $y(n)$ depend on his productivity parameter $n$. An allocation is feasible if

$$
\int_{N} c(n) d F(n) \leq \int_{N} y(n) d F(n),
$$

so aggregate consumption does not exceed aggregate production. The allocation is incentive compatible if

$$
u\left(c\left(n^{\prime}\right), y\left(n^{\prime}\right), n^{\prime}\right) \geq u\left(c(n), y(n), n^{\prime}\right)
$$


for all $n$ and $n^{\prime}$ in $N$, so nobody has an incentive to claim that his productivity parameter is $n$ when in fact it is $n^{\prime}$. An individual's productivity parameter and labour input are taken to be private information, so incentive compatibility is a prerequisite for the implementation of an allocation.

Allocations are assessed according to the utilitarian welfare functional

$$
\int_{N} v(n) d F(n)
$$

where, for each $n$,

$$
v(n)=u(c(n), y(n), n)
$$

is the utility that a person with productivity parameter $n$ obtains from the allocation. The utilitarian welfare maximization problem is to maximize (2.4) over the set of feasible and incentive compatible allocations. By the taxation principle of Hammond (1979) and Guesnerie (1995), this problem is equivalent to the problem of choosing an optimal tax schedule $T(\cdot)$ and then letting each person choose an output provision level $y$ and a consumption level $c=y-T(y)$. Therefore, I refer to it as the optimal income tax problem.

As discussed in the introduction, I will not study the optimal income tax problem directly. Following a line of argument that was first developed by Matthews and Moore (1987) for a monopoly problem, I will instead study the modified problem which is obtained if the requirement of incentive compatibility is weakened to downward incentive compatibility and, in addition, a weak monotonicity requirement is imposed on consumption. I refer to this modified problem as the weakly relaxed income tax problem. The word "weakly" highlights the fact that I do not just drop the upward incentive constraints, but that I replace them with a monotonicity constraint on consumption. ${ }^{8}$

In the weakly relaxed income tax problem, an allocation $(c(\cdot), y(\cdot))$ is chosen to maximize (2.4) subject to the feasibility constraint (2.2) and the requirement that

$$
u\left(c\left(n^{\prime}\right), y\left(n^{\prime}\right), n^{\prime}\right) \geq u\left(c(n), y(n), n^{\prime}\right)
$$

and

$$
c\left(n^{\prime}\right) \geq c(n)
$$

\footnotetext{
${ }^{8}$ In contrast, Matthews and Moore (1987) study a relaxed problem involving only downward incentive constraints. In their general discussion of how to simplify global incentive constraints, they mention the possibility of using monotonicity in combination with adjacent downward incentive compatibility; see in particular fn. 15, p. 447. Their discussion is taken up again by Besley and Coate (1995, p. 197).
} 
for all $n$ and all $n^{\prime} \geq n$ in $N$. I will show that, under standard assumptions, this problem is actually equivalent to the optimal income tax problem, namely that both problems have the same solutions. This is useful because, in the weakly relaxed income tax problem, the implications of redistribution concerns and incentive constraints are easier to disentangle than in the optimal income tax problem itself.

I restrict the analysis to canonical solutions. A solution $(c(\cdot), y(\cdot))$ to the weakly relaxed or the optimal income tax problem is called canonical if it is continuous at any endpoint of $N$ to which the distribution $F$ assigns zero mass. By focussing on canonical solutions, I avoid having to account for the trivial multiplicity of equivalent solutions which arises because modifications of an allocation on null sets have no effects on the value of the welfare functional (2.4) or the validity of the feasibility constraint (2.2) and, moreover, at the endpoints $n^{0}=\min N$ and $n^{1}=\max N$, such modifications are possible without upsetting downward incentive compatibility or incentive compatibility.

The restriction to canonical allocation involves no significant loss of generality. Indeed, if $N$ is finite, it involves no loss of generality at all. If $N$ is an interval, then, for any non-canonical solution of the weakly relaxed or the optimal income tax problem, there an equivalent canonical solution can always be obtained by replacing $(c(n), y(n))$ at any endpoint that has zero mass with the corresponding limit of $\left(c\left(n^{\prime}\right), y\left(n^{\prime}\right)\right)$ as $n^{\prime}$ converges to the endpoint in question.

Remark 2.1 If $(c(\cdot), y(\cdot))$ is a solution to the weakly relaxed or the optimal income tax problem, an equivalent canonical solution $(\bar{c}(\cdot), \bar{y}(\cdot))$ is obtained by setting $\left(\bar{c}\left(n^{0}\right), \bar{y}\left(n^{0}\right)\right)=\lim _{n \downarrow n^{0}}(c(n), y(n))$ if $F\left(\left\{n_{0}\right\}\right)=0$ and $\left(\bar{c}\left(n^{1}\right), \bar{y}\left(n^{1}\right)\right)=\lim _{n \uparrow n^{1}}(c(n), y(n))$ if $F\left(\left\{n^{1}\right\}\right)=0$, leaving everything else unchanged.

I shall be interested in the efficiency properties of the canonical solutions to the weakly relaxed and the optimal income tax problem. For any $n \in N$, the consumption/output pair $(c(n), y(n))$ is said to be efficient for $n$ if

$$
(c(n), y(n))=\left(c^{*}(n, v(n)), y^{*}(n, v(n))\right),
$$

where, for any $v,\left(c^{*}(n, v), y^{*}(n, v)\right)$ is the pair which provides the person with productivity parameter $n$ with the utility $v$ at the lowest cost in terms of required net resources $c-y$, i.e., $\left(c^{*}(n, v), y^{*}(n, v)\right)$ solves the problem

$$
\min _{u(c, y, n) \geq v}(c-y)
$$


the pair $(c(n), y(n))$ is said to be distorted downward from efficiency if

$$
(c(n), y(n))<<\left(c^{*}(n, v(n)), y^{*}(n, v(n))\right)
$$

and to be distorted upward from efficiency if

$$
(c(n), y(n))>>\left(c^{*}(n, v(n)), y^{*}(n, v(n))\right) .
$$

\subsection{Assumptions}

The following assumptions are imposed throughout the paper.

RMQ Regularity, Monotonicity, and Quasiconcavity: The utility function $u: \Re_{+}^{3} \rightarrow \Re$ is twice continuously differentiable, as well as increasing in $c$, decreasing in $y$, nondecreasing in $n$, and strictly quasiconcave in $c$ and $y$.

PEP Positivity of Efficient Production: For all $n \in N$ and all $v$ in the range of $u(\cdot, \cdot, n)$, there exists a unique pair $\left(c^{*}(n, v), y^{*}(n, v)\right)$ that solves problem (2.9); moreover, $y^{*}(n, v)>0$.

SSCC Strict Single-Crossing Condition: The utility function satisfies

$$
\frac{\partial}{\partial n}\left[\frac{u_{c}(c, y, n)}{\left|u_{y}(c, y, n)\right|}\right]>0
$$

for all $(c, y, n) \in \Re_{++}^{2} \times(0,1)$.

DR Desirability of Redistribution: For any $(c, y, n) \in \Re_{+}^{2} \times\left[n^{0}, n^{1}\right)$, there exists $\varepsilon>0$ such that $n+\varepsilon \in N$, and, for all $n^{\prime} \in(n, n+\varepsilon]$ and all $\left(c^{\prime}, y^{\prime}\right) \in \Re_{+}^{2}$ satisfying $c^{\prime} \geq c$ and $u\left(c^{\prime}, y^{\prime}, n^{\prime}\right) \geq u\left(c, y, n^{\prime}\right)$, the inequality

$$
\left|u_{y}\left(c^{\prime}, y^{\prime}, n^{\prime}\right)\right|<\left|u_{y}(c, y, n)\right|
$$

holds (a) if $c^{\prime}=c$ and $y^{\prime} \leq y$, and (b) if $(c, y)$ is efficient for $n$, and $\left(c^{\prime}, y^{\prime}\right)$ is efficient or distorted downwards from efficiency for $n^{\prime}$.

Assumptions RMQ, PEP, and SSCC are standard. In RMQ, I postulate quasiconcavity rather than concavity of $u$. As indicated by Proposition 2.2 below, concavity can play a role in the desirability of redistribution, but then, it belongs in the context of DR. PEP means that, for every $n$ and and every attainable utility level $v$, the efficient pair $\left(c^{*}(n, v), y^{*}(n, v)\right)$ is well 
defined and unique; moreover, the efficient output level $y^{*}(n, v)$ is strictly positive. SSCC reflects the notion that the tradeoff between consumption and leisure results in a higher level of consumption and a lower level of leisure (more output provision) when the productivity parameter is higher. As discussed, e.g., in Milgrom and Shannon (1994), under RMQ, SSCC implies that, for all $(c, y),\left(c^{\prime}, y^{\prime}\right)$ in $\Re_{+}^{2}$ and all $n, n^{\prime}$ in $\Re_{+}$, the inequalities $u(c, y, n) \geq u\left(c^{\prime}, y^{\prime}, n\right), y>y^{\prime}$, and $n>n^{\prime}$ imply $u(c, y, n)>u\left(c^{\prime}, y^{\prime}, n\right) .^{9}$

Condition DR provides the rationale for utilitarian redistribution. If an allocation $(c(\cdot), y(\cdot))$ satisfies the inequality $(2.13)$ for some $n$ and $n^{\prime}$ in $N$, then, at this allocation, people with productivity parameter $n^{\prime}$ have a lower marginal disutility of providing additional output than people with productivity parameter $n$. The sum of these people's utilities is therefore increased if output requirements are redistributed from people with productivity parameter $n$ to people with productivity parameter $n^{\prime}$. If there are no incentive considerations standing in the way, the utilitarian welfare maximizer would like to redistribute output requirements so as to allow the people with productivity parameter $n$ to enjoy more and the people with productivity parameter $n^{\prime}$ to enjoy less leisure.

Condition DR postulates this desirability of redistributing output requirements for two types of allocations. Part (a) postulates it for allocations satisfying $c\left(n^{\prime}\right)=c(n)$ and $y\left(n^{\prime}\right) \leq y(n)$ where $n^{\prime}>n$ is close to $n$. In combination with downward incentive compatibility, this postulate will be used to show that, at a solution to the weakly relaxed income tax problem, people with the same consumption must also produce the same output. For the utility specification (2.1) with concave $U$, this property holds automatically because people with type $n^{\prime}$ have to work fewer hours to provide any given output than people with type $n<n^{\prime}$.

Part (b) of DR postulates the desirability of redistributing output requirements for allocations where $(c(n), y(n))$ is efficient for $n,\left(c\left(n^{\prime}\right), y\left(n^{\prime}\right)\right)$ is efficient or distorted downward from efficiency for $n^{\prime}$, and $n^{\prime}>n$ is close to $n$. This postulate will be used to show that, at any solution to the weakly relaxed income tax problem, unless $n=n^{0}$ and $F\left(\left\{n^{0}\right\}\right)=0$, the pair

\footnotetext{
${ }^{9}$ If $N$ is a finite set, the strictness of the inequality in (2.12) is only needed near the top; below the second-highest type, it is enough to have a weak inequality in (2.12). If $N$ is an interval, this weakening is not possible; equality in (2.12) might imply that types in some subinterval are observationally indistinguishable. The model then is equivalent to one where the distribution of types has a mass point, and the result in Hellwig (2005 b) implies that, unless there is additional bunching, a zero marginal tax is optimal.
} 


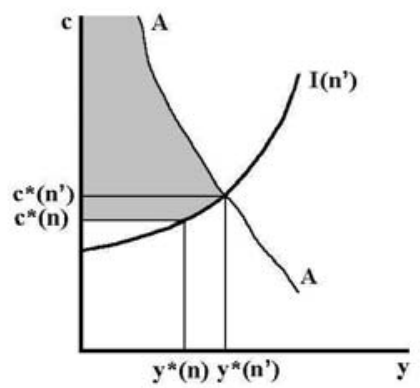

Figure 1: The set of outcome pairs $\left(c^{\prime}, y^{\prime}\right)$ for $n^{\prime}$ to which part (b) of DR applies.

$(c(n), y(n))$ cannot be efficient.

The condition is illustrated in Figure 1. In this figure, $\left(c^{*}(n), y^{*}(n)\right)$ is an efficient outcome pair for type $n, I\left(n^{\prime}\right)$ is the indifference curve of type $n^{\prime}>n$ through $\left(c^{*}(n), y^{*}(n)\right)$, and $A-A$ is the locus of efficient outcome pairs for type $n^{\prime}$. Part (b) of DR postulates that, if $n^{\prime}>n$ is sufficently close to $n$, then $(2.13)$ hold for all outcome pairs $\left(c\left(n^{\prime}\right), y\left(n^{\prime}\right)\right)$ in the shaded area to the left of the locus $A-A$ of efficient outcome pairs for type $n^{\prime}$ and on or above the indifference curve $I\left(n^{\prime}\right)$ and the horizontal line through the reference pair $\left(c^{*}(n), y^{*}(n)\right)$.

To appreciate the relation of condition DR to the literature, it is useful to consider the following alternative condition:

GMSC Generalized Mirrlees-Seade Condition: (a) For all $(c, y, n) \in$ $\Re_{+}^{3}, u_{y y}(c, y, n) \leq 0$ and $u_{y n}(c, y, n)>0$, and (b) for any two triples $(c, y, n)$ and $\left(c^{\prime}, y^{\prime}, n^{\prime}\right)$ such that $n^{\prime}>n, c^{\prime} \geq c$, and $u\left(c^{\prime}, y^{\prime}, n^{\prime}\right) \geq$ $u\left(c, y, n^{\prime}\right)$, one has

$$
u_{c}\left(c^{\prime}, y^{\prime}, n^{\prime}\right)<u_{c}(c, y, n) \text { or }\left|u_{y}\left(c^{\prime}, y^{\prime}, n^{\prime}\right)\right|<\left|u_{y}(c, y, n)\right| .
$$

For concave $u$, part (a) of GMSC is the same as part (a) of DR. For utility functions taking the special form (2.1), this condition is automatically satisfied if $u$ is concave. ${ }^{10}$ Part (b) of GMSC corresponds to the "very weak

\footnotetext{
${ }^{10}$ For arbitrary utility specifications, this is not true. For such specifications, GMSC is therefore stronger than Roëll's imposition of concavity and part (b) of GMSC. The
} 
redistribution assumption" that Roëll (1985) gave for the finite-type model of Guesnerie and Seade (1982). This condition differs from part (b) of DR in two respects. First, in GMSC, the condition is formulated in global rather than local terms, referring to all $n^{\prime}>n$, rather than all $n^{\prime}>n$ that are sufficiently close to $n$. Second, part (b) of GMSC asserts the desirability of redistributing required output or consumption. If the premises of this condition are satisfied, then either the marginal utility of consumption or the marginal disutility of working to provide additional output is lower for the person with productivity parameter $n^{\prime}$ than for the person with productivity parameter $n$; the utilitarian welfare maximizer then desires a redistribution of leisure or of consumption from the former to the latter. In contrast, part (b) of DR asserts the desirability of redistributing output requirements. Moreover, this condition compares $u_{y}(c, y, n)$ and $u_{y}\left(c^{\prime}, y^{\prime}, n^{\prime}\right)$ only for types and outcome pairs such that $n$ and $n^{\prime}$ are close to each other, $(c, y)$ efficient for type $n$ and $\left(c^{\prime}, y^{\prime}\right)$ not distorted upwards from efficiency for type $n^{\prime}$.

Because (2.14) allows for an alternative and (2.13) does not, one may be tempted to consider GMSC to be the weaker condition. In fact, the opposite is true. If the additional premises of part (b) of DR are satisfied, i.e., if $(c, y)$ is efficient for $n$ and $\left(c^{\prime}, y^{\prime}\right)$ is efficient or distorted downwards from efficiency for $n^{\prime}$, one has $u_{c}(c, y, n) \leq\left|u_{y}(c, y, n)\right|$ and $u_{c}\left(c^{\prime}, y^{\prime}, n^{\prime}\right) \geq\left|u_{y}\left(c^{\prime}, y^{\prime}, n^{\prime}\right)\right|$, so (2.14) actually implies (2.13). Thus GMSC implies DR.

What does condition DR mean for the utility function $u$ ? For utility functions taking the special form (2.1), GMSC is implied by strict concavity of $u$ in combination with Seade's (1982) assumption that leisure is a noninferior good. ${ }^{11}$ Trivially therefore, for such utility functions, DR is also implied by strict concavity of $u$ and noninferiority of leisure. The following result gives a more general condition for $u$ to satisfy DR. This condition does not presume that $u$ takes the special form (2.1).

Proposition 2.2 Assume RMQ, PEP, and SSCC, and suppose that, for any $n$, the indifference curves of the utility function $u(\cdot, \cdot, n)$ have strictly positive Gaussian curvature. ${ }^{12}$ Assume also that $N$ is an interval. Then condition DR holds if $u$ is concave in $c$ and $y$ and, moreover,

$$
u_{y n}(c, y, n)>0
$$

additional assumption is needed to eliminate the possibility that the weakly relaxed income tax problem has a solution with $c(n)=c\left(n^{\prime}\right)$ and $y(n)>y\left(n^{\prime}\right)$ for some $n$ and $n^{\prime}>n$. For the optimal income tax problem itself, this possibility is eliminated by upward incentive compatibility.

${ }^{11}$ See Roëll (1985), Brunner (1993, 1995), and Homburg (2004).

${ }^{12}$ I.e. that the quadratic forms $u_{y}^{2} u_{c c}-2 u_{c} u_{y} u_{c y}+u_{c}^{2} u_{y y}$ are everywhere strictly negative. 
for all $(c, y, n)$ and

$$
u_{n y}\left(c^{*}(n, v), y^{*}(n, v), n\right) \frac{\partial y^{*}}{\partial v}+u_{n c}\left(c^{*}(n, v), y^{*}(n, v), n\right) \frac{\partial c^{*}}{\partial v}<0,
$$

for all $n \in N$ and all $v$ in the range of $u(\cdot, \cdot, n)$. Under these assumptions on the functions $u, c^{*}$, and $y^{*}$, condition DR also holds if $N$ is a finite set and the differences between neighbouring elements of $N$ are uniformly small.

Proposition 2.2 reduces the desirability of redistribution to three substantive properties of the utility function: First, $u$ is concave. Second, the marginal disutility of producing additional output is decreasing in $n$. Third, for any $n^{\prime} \in N$, the function $v \rightarrow u_{n}\left(c^{*}\left(n^{\prime}, v\right), y^{*}\left(n^{\prime}, v\right), n^{\prime}\right)$ is differentiable, and its slope is negative. Thus, in Figure $1, u_{n}$ is strictly decreasing as one is moving up along the locus $A-A$ of efficient outcome pairs for type $n^{\prime}$.

The first two properties are familiar from the literature. Concavity of $u$ reflects inequality aversion or neutrality. Positivity of $u_{y n}$ reflects the idea that a person with higher productivity has a lower marginal disutility of producing additional output than a person with lower productivity. For the simple utility specification (2.1) with concave $U$, this idea combines the two notions that (i) the person with higher $n$ needs less labour to produce the given output $y$ and therefore has a lower marginal disutility of labour, and (ii) the person with higher $n$ needs a smaller labour increment to obtain a given output increment.

To understand the third property, observe that the locus of efficient outcome pairs coincides with the locus of solutions to the problem of maximizing $u\left(c, y, n^{\prime}\right)$ under the budget constraint $c=\alpha+y$. Let $v^{*}$, given by

$$
v^{*}\left(\alpha, n^{\prime}\right):=\max _{y \geq 0} u\left(\alpha+y, y, n^{\prime}\right)
$$

be the associated indirect utility function. Under the given assumptions, $v^{*}$ is twice continuously differentiable, with $v_{n}^{*}=u_{n}$ and

$$
v_{n \alpha}^{*}=\frac{\partial u_{n}\left(c^{*}\left(n^{\prime}, v\right), y^{*}\left(n^{\prime}, v\right), n^{\prime}\right)}{\partial v} v_{\alpha}^{*} .
$$

Condition (2.16) is therefore equivalent to the requirement that $v_{n \alpha}^{*}\left(\alpha, n^{\prime}\right)<$ 0 for all $n^{\prime}$ and $\alpha$. This requirement in turn is equivalent to the requirement that $v_{\alpha n}^{*}\left(\alpha, n^{\prime}\right)<0$ for all $n^{\prime}$ and $\alpha$, i.e., that, in the absence of distortionary taxation, the "social marginal utility of income" is a decreasing function of $n$. 
This latter condition is again well known. It figures prominently in the literature on optimal utilitarian linear income taxation, see e.g., Hellwig (1986). However, there it is used globally, to assess the welfare implications of raising the marginal income tax everywhere above zero. Here, the condition $v_{\alpha n}<0$ is used locally to establish part (b) of consition DR, i.e., to show that, for any $n \in N$ and any $n^{\prime}>n$ sufficiently close to $n$, the inequality (2.13) is satisfied if the assignment of $(c, y)$ to $n$ and of $\left(c^{\prime}, y^{\prime}\right)$ to $n^{\prime}$ is compatible with consumption monotonicity and downward incentive compatibility and, moreover, $(c, y)$ is efficient for $n$ and $\left(c^{\prime}, y^{\prime}\right)$ is efficient or distorted downwards from efficiency for $n^{\prime}$.

For utility functions taking the form (2.1), the inequality $v_{\alpha n}^{*}<0$ and therefore (2.16) is implied by strict concavity of $u$ and noninferiority of leisure. ${ }^{13}$ For arbitrary utility functions, (2.16) holds if consumption is normal, ${ }^{14}$ if leisure is noninferior, and if $u_{y n}>0$ and $u_{c n}<0$.

Regardless of the signs of $\frac{\partial y^{*}}{\partial v}$ and $\frac{\partial c^{*}}{\partial v}$, i.e., regardless of the ordinal properties of $u$, condition DR is always satisfied if $u$ is sufficiently concave. To see this, suppose that $u=\varphi \circ \hat{u}$, where $\hat{u}$ satisfies RMQ, PEP, and SSCC, and $\varphi$ is twice continuously differentiable, increasing and strictly concave. Then $u$ and $\hat{u}$ have the same ordinal properties. Moreover, conditions (2.15) and (2.16) take the form

$$
\begin{gathered}
\varphi^{\prime} \hat{u}_{y y}+\varphi^{\prime \prime} \hat{u}_{y}^{2} \leq 0 \\
\varphi^{\prime} \hat{u}_{y n}+\varphi^{\prime \prime} \hat{u}_{y} \hat{u}_{n}>0
\end{gathered}
$$

and

$$
\varphi^{\prime}\left[\hat{u}_{n y} \frac{\partial y^{*}}{\partial v}+\hat{u}_{n c} \frac{\partial c^{*}}{\partial v}\right]+\varphi^{\prime \prime} \hat{u}_{n}<0 .
$$

From these conditions, one immediately obtains:

Corollary 2.3 Assume that $\hat{u}$ satisfies $R M Q, P E P$, and $S S C C$, and suppose that the efficient-outcome function $\left(c^{*}(\cdot, \cdot), y^{*}(\cdot, \cdot)\right)$ is continuously differentiable. Let $\varphi$ be twice continuously differentiable, increasing and strictly concave. If $N$ is an interval, the function $u=\varphi \circ \hat{u}$ satisfies condition $D R$ if the curvature $-\frac{\varphi^{\prime \prime}}{\varphi^{\prime}}$ of the function $\varphi$ is everywhere sufficiently large.

\footnotetext{
${ }^{13}$ See Christiansen (1983) or Werning (2000). For utility functions taking the form (2.1), Roy's identity implies that the indirect utility function $v^{*}$ satisfies $v_{n}^{*}=v_{\alpha}^{*} \frac{y}{n}$. Hence, $v_{\alpha n}^{*}=v_{n \alpha}^{*}=v_{\alpha \alpha}^{*} \frac{y}{n}+v_{\alpha}^{*} \frac{y_{\alpha}}{n}$.

${ }^{14}$ Under RMQ and SSCC, (2.16) is also satisfied if consumption is nonnormal and $u_{y n}>$ 0 .
} 
Corollary 2.3 highlights the importance of the cardinal properties of $u$ for the desirability of redistribution. Whereas, e.g., Seade (1982) used strict concavity, a cardinal property, and noninferiority of leisure, an ordinal property, to derive the desirability of redistribution, Corollary 2.3 shows that, whatever the ordinal properties of $u$ may be, (local) redistribution of leisure is always desirable if $u(\cdot, \cdot, n)$ is sufficiently concave.

This being said, one should also see that, for reasons related to the ordinal properties of $u$, condition DR can be satisfied even if $u$ is not concave. This is the case, e.g., for the linearly homogeneous Cobb-Douglas specification

$$
u(c, y, n)=c^{\beta}\left(1-\frac{y}{n}\right)^{1-\beta} .
$$

This specification satisfies DR. However, the desire for redistributing leisure is driven by the consideration that the disutility of producing additional output is lower for people with higher $n$, rather than by inequality aversion. This observation indicates that, in model with heterogeneous productivity levels, redistributive utilitarian intervention is motivated not only by inequality aversion, but also by the consideration that the total burden of producing a given aggregate output is smaller if output requirements are aligned with productivity parameters.

\section{The Main Theorems}

Throughout the paper, conditions RMC, PEP, SSCC, and DR will be taken for granted without any further mention. Under these assumptions, one can prove the following results.

Theorem 3.1 Assume that the support $N$ of the distribution $F$ is finite or that $N$ is an interval $\left[n^{0}, n^{1}\right]$ and $F$ has a density $f$, which is continuous and strictly positive on $N$. Let $(c(\cdot), y(\cdot))$ be any canonical solution to the weakly relaxed income tax problem, and let $v(\cdot)$ be the associated indirect utility function. Then $(c(\cdot), y(\cdot))$ and $v(\cdot)$ satisfy the following:

a: There is no distortion at the top, i.e.,

$$
\left(c\left(n^{1}\right), y\left(n^{1}\right)\right)=\left(c^{*}\left(n^{1}, v\left(n^{1}\right)\right), y^{*}\left(n^{1}, v\left(n^{1}\right)\right)\right) .
$$

b: Between the bottom and the top of $N$, i.e., for $n \in\left(n^{0}, n^{1}\right)$, the pair $(c(n), y(n))$ is distorted downward from efficiency and satisfies

$$
(c(n), y(n))<<\left(c^{*}(n, v(n)), y^{*}(n, v(n))\right) ;
$$


indeed, on any compact subset of $\left(n^{0}, n^{1}\right),(c(n), y(n))$ is bounded away from efficiency.

c: At the bottom, i.e., for $n=n^{0}$, consumption-output pairs are also distorted downward from efficiency if the $N$ is a finite set or if the monotonicity constraint on $c(\cdot)$ is strictly binding at $n^{0}$. If $F$ has a density on $N=\left[n^{0}, n^{1}\right]$ and if $c(\cdot)$ is strictly increasing at $n=n^{0},{ }^{15}$ there is no distortion at the bottom, i.e.,

$$
\left(c\left(n^{0}\right), y\left(n^{0}\right)\right)=\left(c^{*}\left(n^{0}, v\left(n^{0}\right)\right), y^{*}\left(n^{0}, v\left(n^{0}\right)\right)\right) .
$$

$d$ : The functions $c(\cdot)$ and $y(\cdot)$ are co-monotonic on $N$, i.e., for any $n$ and $n^{\prime}>n$ in $N, y\left(n^{\prime}\right)>y(n)$ if $c\left(n^{\prime}\right)>c(n)$, and $y\left(n^{\prime}\right)=y(n)$ if $c\left(n^{\prime}\right)=c(n)$. Moreover, the allocation $(c(\cdot), y(\cdot))$ is incentive compatible.

$e$ : The function $y(\cdot)-c(\cdot)$ is co-monotonic with $c(\cdot)$ and $y(\cdot)$. Moreover, there exists $\bar{n} \in\left(n^{0}, n^{1}\right)$ such that $y(n)<c(n)$ for $n \in\left[n^{0}, \bar{n}\right)$ and $y(n)>$ $c(n)$ for $n \in\left(\bar{n}, n^{1}\right]$; in particular, $c\left(n^{0}\right)>y\left(n^{0}\right) \geq 0$.

Theorem 3.2 Under the assumptions of Theorem 3.1, the weakly relaxed income tax problem and the optimal income tax problem have the same canonical solutions. In particular, any canonical solution $(c(\cdot), y(\cdot))$ to the optimal income tax problem satisfies statements (a) - (e) of Theorem 3.1.

Theorem 3.3 Under the assumptions of Theorem 3.1, any tax schedule $T$ that is associated with a canonical solution to the weakly relaxed and the optimal income tax problem is strictly increasing on the range $y(N)$ of the output provision function. If $T$ is differentiable from the right, ${ }^{16}$ the righthand derivative $\tau(\cdot)$ satisfies

$$
\lim _{n \uparrow n_{1}} \tau(y(n))=0,
$$

as well as

$$
\tau(y(n)) \in(0,1)
$$

\footnotetext{
${ }^{15} \mathrm{~A}$ nondecreasing function $g($.$) is said to be strictly increasing at n$ if, for every $\varepsilon>0$, one has $g(n+\varepsilon)>g(n)$ or $g(n)>g(n-\varepsilon)$.

${ }^{16}$ If $N$ is an interval and the allocation $(c(\cdot), y(\cdot))$ is continuous, the tax schedule $T(\cdot)$ is necessarily differentiable on $y(N)$; its derivative $\tau(\cdot)$ is then given by (3.10) holding with equality. However, if $N$ is a finite set or if the allocation $(c(\cdot), y(\cdot))$ is not continuous, the set $y(N)$ is not an interval. In this case, the specification of $T(y)$ for $y \notin y(N)$ is somewhat arbitrary; this arbitrariness introduces the possibility that, at a boundary point of $y(N)$, the specified tax schedule may not be differentiable from the right. However, it is always possible to specify $T(\cdot)$ so that the right-hand derivative $\tau(y)$ exists for all $y$ and satisfies (3.10) for all $n \in N$.
} 
for all $n \in\left(n^{0}, n^{1}\right)$; indeed, on any compact subset of $\left(n^{0}, n^{1}\right), \tau(y(n))$ is bounded away from zero and one. If $F$ has a density on $N=\left[n^{0}, n^{1}\right]$ and if $c(\cdot)$ is strictly increasing at $n=n^{0}$,

$$
\lim _{n \downarrow n^{0}} \tau(y(n))=0 ;
$$

if $N$ is finite or if the monotonicity constraint on $c(\cdot)$ is strictly binding at $n^{0}$, then

$$
\lim _{n \downarrow n^{0}} \tau(y(n))>0
$$

Theorem 3.1 characterizes the solutions to the weakly relaxed income tax problem. Theorem 3.2 establishes the equivalence of the weakly relaxed income tax problem with the optimal income tax problem. Theorem 3.3 formulates the implications of Theorems 3.1 and 3.2 for optimal tax schedules, showing that the optimal marginal income tax is zero at the top and strictly positive between the top and the bottom; indeed, on any compact subset of $\left(n^{0}, n^{1}\right)$, the optimal marginal tax is bounded away from zero. At $y\left(n^{0}\right)$, the optimal marginal income tax is positive if the fraction of the population producing the output $y\left(n^{0}\right)$ is positive (because $n^{0}$ has positive mass or because there is bunching). The optimal marginal income tax at $y\left(n^{0}\right)$ is zero if $F\left(n^{0}\right)=0$, and there is no bunching at $y\left(n^{0}\right)$.

Theorems 3.2 and 3.3 follow in a straightforward manner from Theorem 3.1. Without going into details, I briefly sketch the arguments. For Theorem 3.2 , one notes that the constraint set for the optimal income tax problem is a subset of the constraint set for the weakly relaxed income tax problem. By Statement (d) of Theorem 3.1, any solution to the weakly relaxed income tax problem is incentive compatible and therefore lies in the constraint set for the optimal income tax problem. Such a solution must therefore also be a solution to the optimal income tax problem. Moreover, because all solutions to the optimal income tax problem generate the same welfare level, any other solution to the optimal income tax problem generates the same welfare level as a solution to the weakly relaxed income tax problem and must itself be a solution to the latter problem. Given Theorem 3.1, these considerations establish Theorem 3.2.

As for Theorem 3.3, a tax schedule that implements the allocation $(c(\cdot), y(\cdot))$ must satisfy

$$
T(y(n)):=y(n)-c(n)
$$


for all $n \in N .{ }^{17}$ Incentive compatibility requires that, for all $n \in N, y(n)$ maximizes $u(y-T(y), y, n)$. If $T(\cdot)$ has a right-hand derivative $\tau(y(n))$ at $y(n)$, the first-order condition for this maximization is

$$
u_{c}(c(n), y(n), n)(1-\tau(y(n)))+u_{y}(c(n), y(n), n) \leq 0 .
$$

By a rearrangement of terms, this implies

$$
\tau(y(n)) \geq \frac{u_{c}(c(n), y(n), n)+u_{y}(c(n), y(n), n)}{u_{c}(c(n), y(n), n)},
$$

so $\tau(y(n))$ is zero, positive, or negative as $(c(n), y(n))$ is efficient, distorted downward, or distorted upward from efficiency. Equations (3.4) - (3.7) thus follow from statements (a) - (c) of Theorem 3.1. As for the first statement of Theorem 3.3, this follows directly from (3.8) and statement (e) of Theorem 3.1 .

The argument for Theorem 3.1 is less straightforward. It involves the following major steps. First, if consumption is constant on some set of types, then output requirements must also be constant on this set. If they were not constant, then, by downward incentive compatibility, they would have to be decreasing in $n$. However, by RMQ and part (a) of DR, it would then be possible to raise welfare by equalizing output requirements for the productivity parameters in question. The change could be executed without affecting adjacent downward incentive compatibility.

Second, because upward incentive constraints play no role, outcome pairs cannot be distorted upward from efficiency. If, for type $n$, they were distorted upward, then it would be possible to reduce this type's consumption $c(n)$ and output provision $y(n)$ in such a way that the difference $c(n)-y(n)$ is reduced even though the utility $v(n)=u(c(n), y(n), n)$ is unchanged. By SSCC, such a reduction would not affect downward incentive compatibility. The decrease in $c(n)-y(n)$ could be used to make, e.g., types near the top better off without affecting downward incentive compatibility. Thus, the initial allocation could not have been a solution to the weakly relaxed income tax problem.

Third, at any $n$ between the bottom and the top, either consumption monotonicity or downward incentive compatibility is strictly binding. ${ }^{18}$ If

\footnotetext{
${ }^{17}$ If there is bunching, with $y(n)=\bar{y}$ for several types $n$, incentive compatibility dictates that $c^{A}(n)=\bar{c}$ for the same types, so (3.8) is still unambigous.

${ }^{18}$ In the sense that the corresponding Kuhn-Tucker multipliers or Pontryagin costate variables are nonzero. This is stronger than merely having one of the constraints hold as an equation.
} 
neither constraint were strictly binding, the consumption-output pair assigned to type $n$ would be efficient. Because, by the previous argument, the consumption-output pairs for higher types are efficient or distorted downward from efficiency, part (b) of condition DR would imply that the sum of utilities could be increased by redistributing output requirements from type $n$ to some slightly higher types, making type $n$ better off and the higher types worse off, without affecting adjacent downward incentive compatibility.

Fourth, at any $n^{\prime} \in\left(n^{0}, n^{1}\right)$, some downward incentive compatibility constraint holds as an equation. For suppose that downward incentive compatibility is not binding at $n^{\prime}$. Then, by the preceding argument, consumption monotonicity is strictly binding, i.e., $c(\cdot)$ must be constant in a neighbourhood of $n^{\prime}$. By the first step, $y(\cdot)$ is also constant on this interval. Therefore, for $n^{\prime}$ and $n$ belonging to this interval, the downward incentive compatibility condition (2.6) holds as an equation, i.e., downward incentive compatibility is weakly binding.

Fifth, the functions $c(\cdot)$ and $y(\cdot)$ are co-monotonic, and the allocation is incentive compatible. Given that downward incentive compatibility is everywhere weakly binding, an increase in $c(\cdot)$ must always be accompanied by an increase in $y(\cdot)$ and vice versa. In particular, therefore, the allocation is nondecreasing. Incentive compatibility then follows from the single-crossing condition.

Sixth, $c(n)>0$ for all $n \in N$; in particular, $c\left(n^{0}\right)>0$. Because downward incentive compatibility is everywhere weakly binding, the indifference curve of any type $n \in N$ in the point $(c(n), y(n))$ must be tangent to the image set of the function $(c(\cdot), y(\cdot))$ to the left of this point. In the absence of upward distortions from efficiency, the slope $\frac{d c}{d y}$ of this indifference curve at $(c(n), y(n))$ is no greater than one. Therefore, $c(\cdot)$ cannot be steeper at $n$ than $y(\cdot)$, i.e., the difference $c(\cdot)-y(\cdot)$ must be nonincreasing. Because $c(\cdot)-y(\cdot)$ is nonincreasing, one has $c(n)-y(n) \leq c\left(n^{0}\right)-y\left(n^{0}\right)$ for all $n \in N$. It follows that $c\left(n^{0}\right)>0$ and, by monotonicity, $c(n)>0$ for all $n \in N$. Otherwise, by PEP, all types would be worse off than they are under laissez-faire.

Finally, given that consumption is strictly positive, the same argument as in the third step can be used to show that, for $n \in\left(n^{0}, n^{1}\right)$, the pair $(c(n), y(n))$ must be distorted downward from efficiency. If $(c(n), y(n))$ were efficient, then, with $c(n)>0$ and, by PEP, $y(n)>0$, the pair $(c(n), y(n))$ would have to satisfy the first-order condition

$$
u_{c}(c(n), y(n), n)+u_{y}(c(n), y(n), n)=0 .
$$


If (3.11) holds, a small reduction in type $n$ 's consumption and output provision by the same amount would leave the feasibility constraint (2.2) unaffected. This reduction would have no first-order effects on the utility of type $n$. However, it would have a first-order effect on the utility $u\left(c(n), y(n), n^{\prime}\right)$ that type $n^{\prime}>n$ could obtain by imitating type $n$. Therefore, it would make room for some redistribution of output requirements from type $n$ to type $n^{\prime}$. By part (b) of DR, the overall effect of these changes would be to raise the sum of utilities, contrary to the presumed optimality of the given allocation. Given that $(c(n), y(n))$ is distorted downward from efficiency for $n \in\left(n^{0}, n^{1}\right)$, the argument in the preceding step can be strengthened to the effect that $c(n)-y(n)$ is decreasing at any $n$ at which $c(\cdot)$ and $y(\cdot)$ are increasing.

\section{The Case of Finitely Many Types}

In this section, I prove Theorem 3.1 for the case when the support $N$ of the type distribution $F$ is finite. Without loss of generality, I set $N=$ $\left\{n_{1}, n_{2}, \ldots, n_{m}\right\}$, where $n^{0}=n_{1}<\ldots<n_{m}=n^{1}$. I also write $f_{i}:=F\left(\left\{n_{i}\right\}\right)>$ 0 . An allocation $(c(\cdot), y(\cdot))$ is identified with a sequence $\left\{\left(c_{i}, y_{i}\right)\right\}_{i=1}^{m}$ such that $\left(c_{i}, y_{i}\right)=\left(c\left(n_{i}\right), y\left(n_{i}\right)\right)$ for $i=1, \ldots, m$.

I will not actually study the weakly relaxed income tax problem as such. Instead, I study the problem of choosing $\left\{\left(c_{i}, y_{i}\right)\right\}_{i=1}^{m}$ to maximize

$$
\max _{\left\{\left(c_{i}, y_{i}\right)\right\}_{i=1}^{m}} \sum_{i=1}^{m} u\left(c_{i}, y_{i}, n_{i}\right) f_{i}
$$

subject to the feasibility condition

$$
\sum_{i=1}^{m}\left(y_{i}-c_{i}\right) \geq 0
$$

and the requirement that the adjacent downward incentive constraint

$$
u\left(c_{i}, y_{i}, n_{i}\right) \geq u\left(c_{i-1}, y_{i-1}, n_{i}\right),
$$

and the monotonicity condition

$$
c_{i} \geq c_{i-1}
$$

be satisfied for $i=2, \ldots, m$. In this formulation, the downward incentive constraints (2.6) have been replaced by the adjacent downward incentive 
constraints (4.3). I will show that the conclusions of Theorem 3.1 hold for any solution to this maximization problem. By the same argument as in the proof of Theorem 3.2, it follows that the solutions to the weakly relaxed income tax problem coincide with the solutions to the problem of maximizing (4.1) subject to (4.2) - (4.4) and that these solutions satisfy statements (a) - (e) of Theorem 3.1.

The Lagrangian for problem (4.1) can be written as

$$
\begin{aligned}
& \sum_{i=1}^{m} u\left(c_{i}, y_{i}, n_{i}\right) f_{i}+\lambda \sum_{i=1}^{m}\left(y_{i}-c_{i}\right) f_{i} \\
& +\sum_{i=2}^{m} \mu_{i}\left[u\left(c_{i}, y_{i}, n_{i}\right)-u\left(c_{i-1}, y_{i-1}, n_{i}\right)\right]+\sum_{i=2}^{m} \nu_{i}\left(c_{i}-c_{i-1}\right),
\end{aligned}
$$

where $\lambda$ and $\mu_{i}, \nu_{i}, i=2, \ldots m$, are nonnegative multipliers for the constraints (2.2), (2.6), and (2.7). The Kuhn-Tucker conditions for a solution are:

$$
u_{c}\left(c_{i}, y_{i}, n_{i}\right)\left(f_{i}+\mu_{i}\right)-\lambda f_{i}-\mu_{i+1} u_{c}\left(c_{i}, y_{i}, n_{i+1}\right)+\nu_{i}-\nu_{i+1} \leq 0
$$

for $c_{i}, i=1, \ldots, m$, with a strict inequality only if $c_{i}=0$, and

$$
u_{y}\left(c_{i}, y_{i}, n_{i}\right)\left(f_{i}+\mu_{i}\right)+\lambda f_{i}-\mu_{i+1} u_{y}\left(c_{i}, y_{i}, n_{i+1}\right) \leq 0
$$

for $y_{i}, i=1, \ldots, m$, with a strict inequality only if $y_{i}=0 .{ }^{19}$ Moreover,

$$
\begin{gathered}
\lambda \sum_{i=1}^{m}\left(y_{i}-c_{i}\right) f_{i}=0, \\
\mu_{i}\left[u\left(c_{i}, y_{i}, n_{i}\right)-u\left(c_{i-1}, y_{i-1}, n_{i}\right)\right]=0
\end{gathered}
$$

and

$$
\nu_{i}\left(c_{i}-c_{i-1}\right)=0
$$

for $i=2, \ldots, m$. In the remainder of this section, the allocation $\left\{\left(c_{i}, y_{i}\right)\right\}_{i=1}^{m}$ is taken to be a solution to problem $(4.1) ; \lambda, \mu_{i}$, and $\nu_{i}, i=2, \ldots m$, are the associated Kuhn-Tucker multipliers.

If the monotonicity constraint on consumption is binding, then people with the same consumption must also provide the same output. If they didn't, then, by downward incentive compatibility, the "higher" type would be providing less output. Then part (a) of DR would imply that a redistribution of output requirements from the "lower" type to the "higher" type would raise welfare. This is formally shown as:

\footnotetext{
${ }^{19}$ If $i=m,(4.6)$ and (4.7) hold with $\mu_{i+1}=\nu_{i+1}=0$.
} 
Lemma 4.1 For any $k, c_{k}=c_{k-1}$ implies $y_{k}=y_{k-1}$.

Proof. Suppose that the lemma is false, and let $k$ be such that $c_{k}=c_{k-1}$ and $y_{k} \neq y_{k-1}$. Then downward incentive compatibility implies $y_{k}<y_{k-1}$, hence

$$
u\left(c_{k}, y_{k}, n_{k}\right)>u\left(c_{k-1}, y_{k-1}, n_{k}\right) .
$$

By (4.9), it follows that $\mu_{k}=0$. Therefore (4.7) becomes:

$$
u_{y}\left(c_{k-1}, y_{k-1}, n_{k-1}\right)\left(f_{k-1}+\mu_{k-1}\right)+\lambda f_{k-1}=0
$$

for $i=k-1$ and

$$
u_{y}\left(c_{k}, y_{k}, n_{k}\right) f_{k}+\lambda f_{k}-\mu_{k+1} u_{y}\left(c_{k}, y_{k}, n_{j}\right) \leq 0
$$

for $i=k$. From (4.12) and (4.13), one obtains

$$
u_{y}\left(c_{k-1}, y_{k-1}, n_{k-1}\right) \geq-\lambda \geq u_{y}\left(c_{k}, y_{k}, n_{k}\right),
$$

contrary to part (a) of DR. The assumption that $c_{k}=c_{k+1}$ and $y_{k} \neq y_{k+1}$ for some $k$ has thus led to a contradiction and must be false.

The next lemma shows that, for a solution to problem (4.1), distortions from efficiency all go in the same direction. Consumption and output provision are never distorted upward from efficiency. To abbreviate the notation, I write $\left(c_{k}^{*}, y_{k}^{*}\right)$ for the pair $\left(c^{*}\left(n_{k}, v\left(n_{k}\right)\right), y^{*}\left(n_{k}, v\left(n_{k}\right)\right)\right)$ that provides type $n_{k}$ with the utility $v\left(n_{k}\right)=u\left(c_{k}, y_{k}, n_{k}\right)$ at the lowest net resource requirement.

Lemma 4.2 For any $k,\left(c_{k}, y_{k}\right) \leq\left(c_{k}^{*}, y_{k}^{*}\right)$.

Proof. If the lemma is false, one has $c_{k}>c_{k}^{*}$ or $y_{k}>y_{k}^{*}$ for some $k$. Let $\hat{k}$ be the largest index $k$ for which this is the case. Further, let $I(\hat{k})$ be the set of indices $i$ with the same consumption as index $\hat{k}$, and let $\hat{\imath}:=\min I(\hat{k})$. Lemma 4.1 implies $\left(c_{k}, y_{k}\right)=\left(c_{\hat{k}}, y_{\hat{k}}\right)$ for all $k \in I(\hat{k})$. By RMQ and the fact that

$$
u\left(c_{\hat{k}}, y_{\hat{k}}, n_{\hat{k}}\right)=v\left(n_{\hat{k}}\right)=u\left(c_{\hat{k}}^{*}, y_{\hat{k}}^{*}, n_{\hat{k}}\right),
$$

one must actually have $c_{\hat{k}}>c_{\hat{k}}^{*}$ and $y_{\hat{k}}>y_{\hat{k}}^{*}$. It follows that $c_{\hat{k}}$ and $y_{\hat{k}}$ are both strictly positive, so for $k \in I(\hat{k})$, the first-order conditions (4.6) and (4.7) hold as equations. Upon summing these equations from $\hat{\imath}$ to 
$\hat{k}$, cancelling out all terms that refer to adjacent downward incentive and monotonicity constraints for indices between $\hat{\imath}$ and $\hat{k}$, one obtains

$$
\sum_{i=\hat{\imath}}^{\hat{k}}\left[u_{c}\left(c_{\hat{k}}, y_{\hat{k}}, n_{i}\right) f_{i}-\lambda f_{i}\right]+\mu_{\hat{\imath}} u_{c}\left(c_{\hat{k}}, y_{\hat{k}}, n_{\hat{\imath}}\right)-\mu_{\hat{k}+1} u_{c}\left(c_{\hat{k}}, y_{\hat{k}}, n_{\hat{k}+1}\right)+\nu_{\hat{\imath}}-\nu_{\hat{k}+1}=0
$$

and

$$
\sum_{i=\hat{\imath}}^{\hat{k}}\left[u_{y}\left(c_{\hat{k}}, y_{\hat{k}}, n_{i}\right) f_{i}+\lambda f_{i}\right]+\mu_{\hat{\imath}} u_{y}\left(c_{\hat{k}}, y_{\hat{k}}, n_{\hat{\imath}}\right)-\mu_{\hat{k}+1} u_{y}\left(c_{\hat{k}}, y_{\hat{k}}, n_{\hat{k}+1}\right)=0 .
$$

Upon adding (4.15) and (4.16) and rearranging terms, noting that, by the definition of $\hat{\imath}$ and (4.10), $\nu_{\hat{\imath}}$ must be equal to zero, one further obtains

$$
\begin{gathered}
\sum_{i=\hat{\imath}}^{\hat{k}}\left[u_{c}\left(c_{\hat{k}}, y_{\hat{k}}, n_{i}\right)+u_{y}\left(c_{\hat{k}}, y_{\hat{k}}, n_{i}\right)\right] f_{i}+\mu_{\hat{\imath}}\left[u_{c}\left(c_{\hat{k}}, y_{\hat{k}}, n_{\hat{\imath}}\right)+u_{y}\left(c_{\hat{k}}, y_{\hat{k}}, n_{\hat{\imath}}\right)\right] \\
=\mu_{\hat{k}+1}\left[u_{c}\left(c_{\hat{k}}, y_{\hat{k}}, n_{\hat{k}+1}\right)+u_{y}\left(c_{\hat{k}}, y_{\hat{k}}, n_{\hat{k}+1}\right)\right]+\nu_{\hat{k}+1} .
\end{gathered}
$$

Because $c_{\hat{k}}>0$ and $y_{\hat{k}}>0,\left(c_{\hat{k}}, y_{\hat{k}}\right)>>\left(c_{\hat{k}}^{*}, y_{\hat{k}}^{*}\right)$ implies $u_{c}\left(c_{\hat{k}}, y_{\hat{k}}, n_{\hat{k}}\right)+$ $u_{y}\left(c_{\hat{k}}, y_{\hat{k}}, n_{\hat{k}}\right)<0$. By SSCC, it follows that

$$
u_{c}\left(c_{\hat{k}}, y_{\hat{k}}, n_{i}\right)+u_{y}\left(c_{\hat{k}}, y_{\hat{k}}, n_{i}\right)<0
$$

for all $i<\hat{k}$. Therefore the left-hand side of (4.17) is strictly negative. Because $\mu_{\hat{k}+1}$ and $\nu_{\hat{k}+1}$ are both nonnegative, it follows that (4.17) implies $\mu_{\hat{k}+1}>0$ and

$$
u_{c}\left(c_{\hat{k}}, y_{\hat{k}}, n_{\hat{k}+1}\right)+u_{y}\left(c_{\hat{k}}, y_{\hat{k}}, n_{\hat{k}+1}\right)<0 .
$$

Now $\mu_{\hat{k}+1}>0$ implies $\hat{k}<m$ and, by $(4.9)$,

$$
u\left(c_{\hat{k}+1}, y_{\hat{k}+1}, n_{\hat{k}+1}\right)=u\left(c_{\hat{k}}, y_{\hat{k}}, n_{\hat{k}+1}\right) .
$$

Because $u$ is quasiconcave and $c_{\hat{k}+1} \geq c_{\hat{k}}$, (4.19) and (4.20) imply that $u_{c}\left(c_{\hat{k}+1}, y_{\hat{k}+1}, n_{\hat{k}+1}\right)+u_{y}\left(c_{\hat{k}}, y_{\hat{k}}, n_{\hat{k}+1}\right)<0$, hence

$$
\left(c_{\hat{k}+1}, y_{\hat{k}+1}\right)>>\left(c_{\hat{k}+1}^{*}, y_{\hat{k}+1}^{*}\right),
$$

contrary to the definition of $\hat{k}$ as the largest index for which the assertion of the lemma is false. The assumption that $c_{k}>c_{k}^{*}$ or $y_{k}>y_{k}^{*}$ for some $k$ has thus led to a contradiction and must be false. 
The following lemma shows that, for any $k$ below the top, either the downward incentive compatibility constraint or the consumption monotonicity constraint of type $k+1$ must be strictly binding. Part (b) of DR is crucial for this result.

Lemma 4.3 For any $k<m$, at least one of the multipliers $\mu_{k+1}, \nu_{k+1}$ is nonzero.

Proof. If the lemma is false, then, for some $\hat{k}<m$, one has $\mu_{\hat{k}+1}=$ $\nu_{\hat{k}+1}=0$. For $i=\hat{k}$, the first-order conditions (4.6) and (4.7) become

$$
u_{c}\left(c_{\hat{k}}, y_{\hat{k}}, n_{\hat{k}}\right)\left(f_{\hat{k}}+\mu_{\hat{k}}\right)-\lambda f_{\hat{k}}+\nu_{\hat{k}} \leq 0,
$$

with equality unless $c_{\hat{k}}=0$, and

$$
u_{y}\left(c_{\hat{k}}, y_{\hat{k}}, n_{\hat{k}}\right)\left(f_{\hat{k}}+\mu_{\hat{k}}\right)+\lambda f_{\hat{k}} \leq 0
$$

with equality unless $y_{\hat{k}}=0$. Upon adding these equations, one obtains

$$
\left[u_{c}\left(c_{\hat{k}}, y_{\hat{k}}, n_{\hat{k}}\right)+u_{y}\left(c_{\hat{k}}, y_{\hat{k}}, n_{\hat{k}}\right)\right]\left(f_{\hat{k}}+\mu_{\hat{k}}\right)+\nu_{\hat{k}} \leq 0,
$$

with equality unless $c_{\hat{k}}=0$ or $y_{\hat{k}}=0$. By RMQ and the nonnegativity of $\nu_{\hat{k}}$, it follows that $\left(c_{\hat{k}}, y_{\hat{k}}\right) \geq\left(c_{\hat{k}}^{*}, y_{\hat{k}}^{*}\right)$. By Lemma 4.2 , therefore, $\left(c_{\hat{k}}, y_{\hat{k}}\right)=$ $\left(c_{\hat{k}}^{*}, y_{\hat{k}}^{*}\right)$.

By PEP, it follows that $y_{\hat{k}}>0$. Therefore, (4.22) holds as an equation. This yields:

$$
u_{y}\left(c_{\hat{k}}, y_{\hat{k}}, n_{\hat{k}}\right) \geq-\lambda .
$$

Further, because $\mu_{\hat{k}+1}=0$ and $\mu_{\hat{k}+2} u_{y}\left(c_{\hat{k}+1}, y_{\hat{k}+1}, n_{\hat{k}+2}\right) \leq 0$, the first-order condition (4.7) for $i=\hat{k}+1$ yields

$$
u_{y}\left(c_{\hat{k}+1}, y_{\hat{k}+1}, n_{\hat{k}+1}\right) \leq-\lambda .
$$

Upon combining (4.24) and (4.25), one obtains

$$
\left|u_{y}\left(c_{\hat{k}+1}, y_{\hat{k}+1}, n_{\hat{k}+1}\right)\right| \geq\left|u_{y}\left(c_{\hat{k}}, y_{\hat{k}}, n_{\hat{k}}\right)\right| .
$$

However, because $u_{y}\left(c_{\hat{k}+1}, y_{\hat{k}+1}, n_{\hat{k}+1}\right) \geq u_{y}\left(c_{\hat{k}}, y_{\hat{k}}, n_{\hat{k}+1}\right), c_{\hat{k}+1} \geq c_{\hat{k}}$, and, by Lemma 4.2, $\left(c_{\hat{k}+1}, y_{\hat{k}+1}\right) \leq\left(c_{\hat{k}+1}^{*}, y_{\hat{k}+1}^{*}\right)$, for $i=\hat{k}+1$, part (b) of condition DR implies

$$
\left|u_{y}\left(c_{\hat{k}+1}, y_{\hat{k}+1}, n_{\hat{k}+1}\right)\right|<\left|u_{y}\left(c_{\hat{k}}, y_{\hat{k}}, n_{\hat{k}}\right)\right| .
$$


The assumption that $\mu_{\hat{k}+1}=\nu_{\hat{k}+1}=0$ for some $\hat{k}<m$ has thus led to a contradiction and must be false.

On the basis of Lemma 4.3, one easily finds that adjacent downward incentive constraints are everywhere binding. ${ }^{20}$

Lemma 4.4 For any $k<m$,

$$
v\left(n_{k+1}\right)=u\left(c_{k}, y_{k}, n_{k+1}\right),
$$

Proof. If the lemma is false, there exists $\hat{k}<m$, such that

$$
v\left(n_{\hat{k}+1}\right)>u\left(c_{\hat{k}}, y_{\hat{k}}, n_{\hat{k}+1}\right) .
$$

(The reverse inequality is ruled out by downward incentive compatibility.) Because $v\left(n_{\hat{k}+1}\right)=u\left(c_{\hat{k}+1}, y_{\hat{k}+1}, n_{\hat{k}+1}\right)$, one also has $\left(c_{\hat{k}+1}, y_{\hat{k}+1}\right) \neq\left(c_{\hat{k}}, y_{\hat{k}}\right)$. By Lemma 4.1 and monotonicity, therefore, $c_{\hat{k}+1}>c_{\hat{k}}$. Then (4.9) and (4.10) yield $\mu_{\hat{k}+1}=0$ and $\nu_{\hat{k}+1}=0$. By Lemma 4.3., this is impossible.

Lemma 4.5 The sequences $\left\{c_{i}\right\}_{i=1}^{m}$ and $\left\{y_{i}\right\}_{i=1}^{m}$ are co-monotonic, i.e., for any $k<m, y_{k} \leq y_{k+1}$, with equality if and only if $c_{k}=c_{k+1}$. Moreover, the allocation $\left\{\left(c_{i}, y_{i}\right)\right\}_{i=1}^{m}$ is incentive compatible.

Proof. Under RMQ, the first statement follows trivially from (4.28) and the inequality $c_{k+1} \geq c_{k}$. Because $\left(c_{k}, y_{k}\right) \leq\left(c_{k+1}, y_{k+1}\right),(4.28)$ and SSCC imply

$$
v\left(n_{k}\right) \geq u\left(c_{k+1}, y_{k+1}, n_{k}\right) .
$$

The allocation $\left\{\left(c_{i}, y_{i}\right)\right\}_{i=1}^{m}$ thus satisfies adjacent upward as well as adjacent downward incentive compatibility. Given the monotonicity of the allocation and SSCC, overall incentive compatibility follows by standard arguments.

Relying on Lemmas 4.4 and 4.2 , the next two lemmas show that the difference $c_{k}-y_{k}$ is nondecreasing in $k$ and that consumption is everywhere strictly positive. The argument for Lemma 4.6 is familiar from Guesnerie and Seade (1982).

\footnotetext{
${ }^{20}$ Without taking recourse to Lemma 4.3 , (4.28) could also be established by the geometric argument of Guesnerie and Seade (1982), see also Homburg (2004). However, for Lemma 4.9 below, one needs Lemma 4.3 anyway.
} 
Lemma 4.6 For any $k<m$,

$$
c_{k+1}-y_{k+1} \leq c_{k}-y_{k}
$$

moreover, the inequality in (4.31) is strict unless $c_{k+1}=c_{k}$.

Proof. For any $k<m$, Lemma 4.4 implies that, for type $n_{k+1}$, the point $\left(c_{k}, y_{k}\right)$ lies on the indifference curve through $\left(c_{k+1}, y_{k+1}\right)$. By Lemma 4.2 in combination with the strict quasiconcavity of $u$, the slope of this indifference curve at any point $(c, y)$ below $\left(c_{k+1}, y_{k+1}\right)$ is less than one. The lemma follows immediately.

Lemma 4.7 For all $k, c_{k}>0$.

Proof. If the lemma is false, there is a nonempty set $I_{0}$ of indices $k$ such that $c_{k}=0$ for $k \in I_{0}$. I claim that, in this case, the allocation $\left\{\left(c_{i}, y_{i}\right)\right\}_{i=1}^{m}$ is strictly dominated by the laissez-faire allocation $\left\{\left(y_{i}^{L F}, y_{i}^{L F}\right)\right\}_{i=1}^{m}$, where, for any $i, y_{i}^{L F}=\arg \max u\left(y, y, n_{i}\right)$.

By $(2.7), I_{0}$ takes the form $\{1, \ldots, \hat{k}\}$, where $\hat{k}<m$. By RMQ and PEP, one has

$$
u\left(0, y_{k}, n_{k}\right) \leq u\left(0,0, n_{k}\right)<u\left(y_{k}^{L F}, y_{k}^{L F}, n_{k}\right)
$$

for all $k \in I_{0}$. For $k>\hat{k}$, Lemma 4.6 implies $c_{k}-y_{k} \leq c_{\hat{k}+1}-y_{\hat{k}+1}$ and, since $c_{\hat{k}+1}>c_{\hat{k}}, c_{\hat{k}+1}-y_{\hat{k}+1}<-y_{\hat{k}} \leq 0$. By RMQ, it follows that

$$
u\left(c_{k}, y_{k}, n_{k}\right)<u\left(y_{k}^{L F}, y_{k}^{L F}, n_{k}\right)
$$

for $k \notin I_{0}$, as well as for $k \in I_{0}$. However, dominance of the laissez-faire allocation contradicts the assumption that $\left\{\left(c_{i}, y_{i}\right)\right\}_{i=1}^{m}$ is a solution to the weakly relaxed income tax problem. The assumption that $c_{k}=0$ for some $k$ must therefore be false.

Relying on the positivity of consumption, the next lemma shows that, at the top, there is no distortion and the allocation is strictly increasing.

Lemma $4.8\left(c_{m}, y_{m}\right)=\left(c_{m}^{*}, y_{m}^{*}\right)$ and $\left(c_{m-1}, y_{m-1}\right)<<\left(c_{m}, y_{m}\right)$.

Proof. By Lemma 4.7, $c_{m}>0$. In combination with the feasibility constraint (2.2), Lemma 4.7 also implies $y_{k}>0$ for some $k$. By the monotonicity of output provision that was established in Lemma 4.4, it follows that $y_{m}>0$. For $i=m$, therefore, (4.6) and (4.7) hold as equations. Upon 
adding these equations and noting that $\mu_{m+1}=\nu_{m+1}=0$ and $\nu_{m} \geq 0$, one obtains

$$
\left(u_{c}\left(c_{m}, y_{m}, n_{m}\right)+u_{y}\left(c_{m}, y_{m}, n_{m}\right)\right)\left(f_{m}+\mu_{m}\right) \leq 0,
$$

hence $\left(c_{m}, y_{m}\right) \geq\left(c_{m}^{*}, y_{m}^{*}\right)$. By Lemma 4.2 , it follows that $\left(c_{m}, y_{m}\right)=\left(c_{m}^{*}, y_{m}^{*}\right)$.

By SSCC, (4.34) implies $u_{c}\left(c_{m}, y_{m}, n_{m-1}\right)+u_{y}\left(c_{m}, y_{m}, n_{m-1}\right)<0$. By Lemmas 4.2 and 4.4 , it follows that $\left(c_{m-1}, y_{m-1}\right)<<\left(c_{m}, y_{m}\right)$.

To conclude the argument, I finally show that, below the top, consumption and output provision must be distorted downward from efficiency. The argument again relies on Lemma 4.3 and, thereby, on part (b) of condition DR.

Lemma 4.9 For any $k<m,\left(c_{k}, y_{k}\right)<<\left(c_{k}^{*}, y_{k}^{*}\right)$.

Proof. Suppose that the lemma is false. Then, for some $k<m$, one has $c_{k}>0$ and $c_{k} \geq c_{k}^{*}$ or $y_{k} \geq y_{k}^{*}$. Let $\hat{k}$ be the largest index for which this is the case. By Lemma $4.2, c_{\hat{k}} \geq c_{\hat{k}}^{*}$ or $y_{\hat{k}} \geq y_{\hat{k}}^{*}$ is only possible if $\left(c_{\hat{k}}, y_{\hat{k}}\right)=\left(c_{\hat{k}}^{*}, y_{\hat{k}}^{*}\right)$. By PEP, it follows that $y_{\hat{k}}>0$. For $i=\hat{k}$, therefore, (4.7) as well as (4.6) must hold as equations. Upon adding these equations, one obtains

$$
\begin{aligned}
& {\left[u_{c}\left(c_{\hat{k}}, y_{\hat{k}}, n_{\hat{k}}\right)+u_{y}\left(c_{\hat{k}}, y_{\hat{k}}, n_{\hat{k}}\right)\right]\left(f_{\hat{k}}+\mu_{\hat{k}}\right)+\nu_{\hat{k}} } \\
= & \mu_{\hat{k}+1}\left[u_{c}\left(c_{\hat{k}}, y_{\hat{k}}, n_{\hat{k}+1}\right)+u_{y}\left(c_{\hat{k}}, y_{\hat{k}}, n_{\hat{k}+1}\right)\right]+\nu_{\hat{k}+1} .
\end{aligned}
$$

With $c_{\hat{k}}>0$ and $y_{\hat{k}}>0$, efficiency of the pair $\left(c_{\hat{k}}, y_{\hat{k}}\right)$ implies

$$
u_{c}\left(c_{\hat{k}}, y_{\hat{k}}, n_{\hat{k}}\right)+u_{y}\left(c_{\hat{k}}, y_{\hat{k}}, n_{\hat{k}}\right)=0
$$

so (4.35) yields

$$
\nu_{\hat{k}}=\mu_{\hat{k}+1}\left[u_{c}\left(c_{\hat{k}}, y_{\hat{k}}, n_{\hat{k}+1}\right)+u_{y}\left(c_{\hat{k}}, y_{\hat{k}}, n_{\hat{k}+1}\right)\right]+\nu_{\hat{k}+1} .
$$

I claim that the left-hand side of (4.37) is zero. For suppose not and let $\nu_{\hat{k}}>0$. By (4.10) and Lemma 4.1, one then has $c_{\hat{k}-1}=c_{\hat{k}}$ and $y_{\hat{k}-1}=y_{\hat{k}}$. At the same time, (4.36) and SSCC imply

$$
u_{c}\left(c_{\hat{k}}, y_{\hat{k}}, n_{i}\right)+u_{y}\left(c_{\hat{k}}, y_{\hat{k}}, n_{i}\right)<0,
$$

so Lemma 4.2 implies $\left(c_{\hat{k}-1}, y_{\hat{k}-1}\right) \neq\left(c_{\hat{k}}, y_{\hat{k}}\right)$ for $i<\hat{k}$. The assumption that $\nu_{\hat{k}}>0$ thus leads to a contradiction and must be false.

Thus, (4.37) implies

$$
\mu_{\hat{k}+1}\left[u_{c}\left(c_{\hat{k}}, y_{\hat{k}}, n_{\hat{k}+1}\right)+u_{y}\left(c_{\hat{k}}, y_{\hat{k}}, n_{\hat{k}+1}\right)\right]+\nu_{\hat{k}+1}=0 .
$$


However, by SSCC, (4.36) also implies $u_{c}\left(c_{\hat{k}}, y_{\hat{k}}, n_{\hat{k}+1}\right)+u_{y}\left(c_{\hat{k}}, y_{\hat{k}}, n_{\hat{k}+1}\right)>$ 0 . Because $\mu_{\hat{k}+1}$ and $\nu_{\hat{k}+1}$ are nonnegative, it follows that (4.38) implies $\mu_{\hat{k}+1}=\nu_{\hat{k}+1}=0$. By Lemma 4.3, this is impossible.

The preceding arguments show that the conclusions of Theorem 3.1 hold for any solution to problem (4.1): Statement (a) follows from Lemma 4.8, statements (b) and (c) from Lemma 4.9, statement (d) from Lemma 4.5, and statement (e) from Lemmas 4.6 and 4.7.

Given that the constraint set for the weakly relaxed income tax problem is a subset of the constraint set for problem (4.1) and, by Lemma 4.5, any solution to the latter problem is incentive compatible, any such solution must also be a solution to the weakly relaxed income tax problem. Moreover, because all solutions to the weakly relaxed income tax problem generate the same welfare level, any other solution to this problem generates the same welfare level as a solution to problem (4.1) and must itself be a solution to the latter problem. The validity of Theorem 3.1 for the case of finitely many types follows immediately.

\section{The Case of a Continuum of Types}

\subsection{The Optimal Control Problem}

The optimal-control problem is formulated in terms of the indirect utility function $v(\cdot)$. If $F$ has a continuous density $f$ on $N=\left[n^{0}, n^{1}\right]$, the welfare functional (2.4) and the feasibility constraint (2.2) take the form

$$
\int_{n^{0}}^{n^{1}} v(n) f(n) d n
$$

and

$$
\int_{n^{0}}^{n^{1}}(y(n)-c(n)) f(n) d n \leq 0 .
$$

By well known arguments, ${ }^{21}$ in the model with a continuum of types satisfying SSCC, the incentive compatibility condition (2.3) is equivalent to the requirement that $v(\cdot)$ satisfy the equation

$$
v(n)=v\left(n^{0}\right)+\int_{n^{0}}^{n} u_{n}\left(c\left(n^{\prime}\right), y\left(n^{\prime}\right), n^{\prime}\right) d n^{\prime}
$$

for all $n \in N$ and that $y(\cdot)$ be nondecreasing on $N$. The following lemma, provides an analogous characterization of downward incentive compatibility.

\footnotetext{
${ }^{21}$ See, e.g., Mirrlees (1976).
} 
Lemma 5.1 An allocation $(c(\cdot), y(\cdot))$ with nondecreasing $c(\cdot)$ is downward incentive compatible if and only if the indirect utility function $v(\cdot)$ that is given by (2.5) satisfies

$$
v(n)=S(n)+\int_{n^{0}}^{n} u_{n}\left(c\left(n^{\prime}\right), y\left(n^{\prime}\right), n^{\prime}\right) d n^{\prime}
$$

for some nondecreasing function $S(\cdot)$.

The proof of Lemma 5.1 is given in the Appendix. The function $S(\cdot)$ can be interpreted as a measure of cumulative slack in downward incentive compatibility conditions. A comparison of (5.4) with (5.5) indicates that a downward incentive compatible and nondecreasing allocation is incentive compatible if and only if this slack function takes the constant value $v\left(n^{0}\right)$.

The weakly relaxed problem is thus equivalent to the problem of choosing $v(\cdot), c(\cdot), y(\cdot), S(\cdot)$ so as to maximize (5.1), subject to $(2.5),(5.2),(5.4)$, and weak monotonicity of $c(\cdot)$ and $S(\cdot)$. If the functions $c(\cdot)$ and $S(\cdot)$ were known to be absolutely continuous, this problem would be actually be a standard problem of optimal control: In this case, one could treat $v(\cdot), c(\cdot)$, and $S(\cdot)$ as state variables and the slopes $q(\cdot):=c^{\prime}(\cdot)$ and $r(\cdot):=S^{\prime}(\cdot)$, as well as the output requirement $y(\cdot)$, as control variables. The downward incentive compatibility condition could be rewritten as

$$
v^{\prime}(n)=r(n)+u_{n}(c(n), y(n), n),
$$

and the slopes $q(n)=c^{\prime}(n)$ and $r(n)=S^{\prime}(n)$ would have to be nonnegative. The Hamiltonian for this control problem would be

$$
\begin{aligned}
H(n)= & v(n) f(n)+\lambda(y-c(n)) f(n)+\chi(n)(u(c(n), y, n)-v(n)) \\
& +\varphi(n)\left[r(n)+u_{n}(c(n), y, n)\right]+\psi(n) q(n),
\end{aligned}
$$

where $\lambda$ and $\chi(n)$ are the Lagrange multipliers of the constraints (5.2) and $(2.5) ; \varphi(\cdot)$ and $\psi(\cdot)$ are the costate variables associated with the state variables $v(\cdot)$ and $c(\cdot)$.

Maximization of the Hamiltonian with respect to the controls requires that for almost every every $n \in N$,

$$
\lambda f(n)+\chi(n) u_{y}(c(n), y(n), n)+\varphi(n) u_{n y}(c(n), y(n), n) \leq 0,
$$

with equality if $y(n)>0$; further,

$$
\varphi(n) \leq 0
$$


with equality if $r(n)>0$, and

$$
\psi(n) \leq 0,
$$

with equality if $q(n)>0$. The costate variables $\varphi(\cdot)$ and $\psi(\cdot)$ must be absolutely continuous, with derivatives satisfying

$$
\varphi^{\prime}(n)=-f(n)+\chi(n)
$$

and

$$
\psi^{\prime}(n) \leq \lambda f(n)-\chi(n) u_{c}(c(n), y(n), n)-\varphi(n) u_{n c}(c(n), y(n), n)
$$

for almost all $n \in N$; (5.11) holds with equality if $c(n)>0$. In addition, $\varphi(\cdot)$ and $\psi(\cdot)$ must satisfy the transversality conditions

$$
\varphi\left(n^{0}\right)=\varphi\left(n^{1}\right)=0,
$$

and

$$
\psi\left(n^{0}\right) c\left(n^{0}\right)=\psi\left(n^{1}\right)=0 .
$$

The Lagrange multiplier $\lambda$ must be nonnegative and must satisfy

$$
\lambda \int_{n^{0}}^{n^{1}}(y(n)-c(n)) f(n) d n=0 .
$$

The argument that I have just sketched, with $c(\cdot)$ and $S(\cdot)$ assumed to be absolutely continuous, corresponds to the approach of Guesnerie and Laffont (1984) or Ebert (1992). In fact, there is no reason to presume that $c(\cdot)$ and $S(\cdot)$ are continuous, let alone absolutely continuous. However, the analysis in Hellwig (2005a) shows that absolute continuity of $c(\cdot)$ and $S(\cdot)$ is not actually needed. Monotonicity itself provides enough structure to permit the formulation of a maximum principle for the given control problem.

The resulting necessary conditions are identical to the ones just given, with one modification: (5.8) must hold as an equation if $S(\cdot)$ is strictly increasing at $n$, and (5.9) must hold as an equation if $c(\cdot)$ is strictly increasing at $n$; these requirements extend the maximum principle for the "slopes" of $c(\cdot)$ and $S(\cdot)$ from the case where these slopes are well defined to the case where these "slopes" may be infinite, e.g., because the functions $c(\cdot)$ and $S(\cdot)$ are discontinuous. Even in this case, the values of the "slopes" that are being chosen must maximize the products of these "slopes" with the costate variables of $c(\cdot)$ and $S(\cdot)$; i.e., if $c(\cdot)$ or $S(\cdot)$ is strictly increasing at $n$, the corresponding costate variable must be zero. 
If one uses (5.10) to eliminate $\chi(n)$ from (5.11) and (5.7), one obtains:

$$
\psi^{\prime}(n)-\lambda f(n)+u_{c} f(n)+\varphi^{\prime}(n) u_{c}+\varphi(n) u_{n c} \leq 0,
$$

with equality if $c(n)>0$, and

$$
\lambda f(n)+u_{y} f(n)+\varphi^{\prime}(n) u_{y}+\varphi(n) u_{n y} \leq 0,
$$

with equality if $y(n)>0$. Except for the fact that $\psi^{\prime}(n)$ appears in the condition for $c(n)$ rather than $y(n)$, these conditions are familiar from Ebert (1992) and Brunner (1993). If $\psi^{\prime}(n)=0$, they reduce to the corresponding conditions in Mirrlees $(1971,1976)$ and Seade $(1977,1982)$.

Upon combining (5.15) and (5.16) so as to eliminate $\varphi^{\prime}(n)$, one further obtains

$$
\psi^{\prime}(n) \leq \lambda \frac{u_{c}+u_{y}}{u_{y}} f(n)-\varphi(n)\left(u_{n c}-\frac{u_{c}}{u_{y}} u_{n y}\right),
$$

with equality if $c(n)>0$ and $y(n)>0$. This is the central condition of the model with a continuous type distribution. If $c(n)>0$ and $y(n)>0$ and $\psi^{\prime}(n)=0$, it yields the equation

$$
\lambda \frac{u_{c}+u_{y}}{u_{y}} f(n)=\varphi(n)\left(u_{n c}-\frac{u_{c}}{u_{y}} u_{n y}\right),
$$

which lies at the core of the analysis of Mirrlees and Seade. Because $u_{y}<0$, the left-hand side is negative or positive, depending on whether $u_{c}+u_{y}$ is positive or negative, i.e., on whether $(c(n), y(n))$ is distorted downward or upward from efficiency. The Single-Crossing Condition implies that $u_{n c}-$ $\frac{u_{c}}{u_{y}} u_{n y}$ is positive, so the sign of the right-hand side is the same as the sign of $\varphi(n)$.

Whereas, at this point, Seade (1982) proceeds to investigate the sign of $\varphi(n)$ by considering the global properties of $\varphi$ when treated as a solution to (5.15) and (5.16), the indirect approach developed here has $\varphi(n) \leq 0$ already from the necessary condition (5.8) for the choice of the slack function $S(\cdot)$. For any $n$ at which (5.18) holds, one immediately knows that $u_{c}+$ $u_{y}$ is nonnegative, i.e., that $(c(n), y(n))$ cannot be distorted upward from efficiency.

Moreover, for any $n, \varphi(n)=0$ implies that $\varphi$ reaches a maximum at $n$. If $\varphi$ were known to be twice continuously differentiable, then for $n \in\left(n^{0}, n^{1}\right)$, $\varphi(n)=0$ would imply that $\varphi^{\prime}(n)=0$ and $\varphi^{\prime \prime}(n) \leq 0$. From (5.18) and from (5.16) holding as an equation, one would therefore obtain $u_{c}+u_{y}=0$ and $\lambda+u_{y}=0$. Moreover, for (5.16) holding as an equation, total differentiation 
would yield $\frac{d}{d n} u_{y}=-\varphi^{\prime \prime}(n) u_{y} \leq 0$. Because this is incompatible with condition DR, it seems that, for $n \in\left(n^{0}, n^{1}\right)$, one cannot have $\varphi(n)=0$. This implies $\varphi(n)<0$ and hence, by (5.18), $u_{c}+u_{y}>0$ so that $(c(n), y(n))$ is distorted downward from efficiency.

Unfortunately, matters are not so simple: One cannot presume that $\varphi$ is twice continuously differentiable, not even that $\varphi^{\prime}$ is continuous. Moreover, one must consider the role of $\psi^{\prime}(n)$ in (5.15) and (5.17) and the possibility that (5.15) - (5.17) are inequalities rather than equations. The formal proof, which is given in the next subsection, addresses these issues.

The proof will follow the same line of argument as the proof for the case of finitely many types. To understand the parallel, it is useful to note that the Hamiltonian (5.6) has the same structure as the sum of terms with index $i$ in the Lagrangian (4.5) for the finite-type problem, except that (5.6) has $\varphi(n)$ and $\psi(n)$ where (4.5) has $-\mu_{i}$ and $-\nu_{i}$. The optimality conditions here have also the same structure as the first-order conditions (4.6) - (4.10). For instance, if one rewrites (4.6) in the form

$\nu_{i}-\nu_{i+1}-\lambda f_{i}+u_{c}\left(c_{i}, y_{i}, n_{i}\right) f_{i}-\left[\mu_{i+1} u_{c}\left(c_{i}, y_{i}, n_{i+1}\right)-\mu_{i} u_{c}\left(c_{i}, y_{i}, n_{i}\right)\right] \leq 0$,

one sees that, with $-\mu_{i}$ and $-\nu_{i}$ taking the place of $\varphi(n)$ and $\psi(n)$, this has the same structure as (5.15). Once the parallel is seen, the line of argument to be followed is clear from the finite-type case.

\subsection{Proof of Theorem 3.1 for a Continuum of Types}

Throughout this subsection, $(c(\cdot), y(\cdot))$ is a canonical solution to the weakly relaxed income tax problem, $v(\cdot)$ is the associated payoff function, and $\lambda \geq 0$, $\chi(\cdot), \varphi(\cdot), \psi(\cdot)$ are the associated Lagrange multipliers and costate variables.

The first step of the proof shows that people with the same consumption must also provide the same output. This corresponds to Lemma 4.1 in the finite-type case. Part (a) of condition DR again plays a central role.

Lemma 5.2 For any $n$ and $n^{\prime}>n$, in $N, c(n)=c\left(n^{\prime}\right)$ implies that $y(\cdot)$ is constant on $\left(n, n^{\prime}\right)$.

Proof. Suppose that the lemma is false, and let $n, n^{\prime}>n$ be such that that $c(n)=c\left(n^{\prime}\right)$, and $y(\cdot)$ is not constant on $\left(n, n^{\prime}\right)$. Because $c(\cdot)$ is constant on $\left[n, n^{\prime}\right]$, downward incentive compatibility implies that $y(\cdot)$ is nonincreasing on $\left[n, n^{\prime}\right]$. Therefore, if $y(\cdot)$ is not constant on $\left(n, n^{\prime}\right)$, there 
exists a point $\hat{n} \in\left(n, n^{\prime}\right)$ at which $y(\cdot)$ is strictly decreasing. Then one has

$$
\begin{aligned}
v\left(n^{k}\right)-v\left(n_{j}\right) & =\int_{n_{j}}^{n^{k}} u_{y}(c(\breve{n}), y(\breve{n}), \breve{n}) d y(\breve{n})+\int_{n_{j}}^{n^{k}} u_{n}(c(\breve{n}), y(\breve{n}), \breve{n}) d \breve{n} \\
& >\int_{n_{j}}^{n^{k}} u_{n}(c(\breve{n}), y(\breve{n}), \breve{n}) d \breve{n},
\end{aligned}
$$

for all $n_{j} \in(n, \hat{n})$ and all $n^{k} \in\left(\hat{n}, n^{\prime}\right)$. The slack function $S(\cdot)$ is therefore strictly increasing at $\hat{n}$. Because (5.8) must hold as an equation if $S(\cdot)$ is strictly increasing at $n$, it follows that $\varphi(\hat{n})=0$. If one defines

$$
h_{y}(n):=\varphi(n) e^{\int_{n_{0}}^{n} \frac{u_{n y}}{u_{y}} d n^{\prime}},
$$

then (5.8) implies $h_{y}(n) \leq 0$ for all $n$, and $\varphi(\hat{n})=0$ implies $h_{y}(\hat{n})=0$, so the function $h_{y}(\cdot)$ has a maximum at $\hat{n}$. For some sequence $\left\{n_{j}\right\}$ which converges to $\hat{n}$ from below, one therefore has $h_{y}^{\prime}\left(n_{j}\right) \geq 0$, hence

$$
\varphi^{\prime}\left(n_{j}\right)+\varphi\left(n_{j}\right) \frac{u_{n y}\left(c\left(n_{j}\right), y\left(n_{j}\right), n_{j}\right)}{u_{y}\left(c\left(n_{j}\right), y\left(n_{j}\right), n_{j}\right)} \geq 0
$$

for all $j$. Moreover, for some sequence $\left\{n^{k}\right\}$ which converges to $\hat{n}$ from above, one has $h_{y}^{\prime}\left(n^{k}\right) \leq 0$, hence

$$
\varphi^{\prime}\left(n^{k}\right)+\varphi\left(n^{k}\right) \frac{u_{n y}\left(c\left(n^{k}\right), y\left(n^{k}\right), n^{k}\right)}{u_{y}\left(c\left(n^{k}\right), y\left(n^{k}\right), n^{k}\right)} \leq 0
$$

for all $k$. Because $y(\cdot)$ is strictly decreasing at $\hat{n}$, one must have $y\left(n_{j}\right)>0$ for all $j$. For $n=n_{j}$, therefore, (5.16) must hold as an equation. Upon combining this equation with (5.20), one concludes that

$$
-\frac{\lambda+u_{y}\left(c\left(n_{j}\right), y\left(n_{j}\right), n_{j}\right)}{u_{y}\left(c\left(n_{j}\right), y\left(n_{j}\right), n_{j}\right)} \geq 0
$$

for all $j$. In contrast, for $n=n^{k},(5.16)$ holds as inequality. Upon combining this inequality with (5.21), taking account of the fact that $u_{y}\left(c\left(n^{k}\right), y\left(n^{k}\right), n^{k}\right)<$ 0 , one finds that

$$
-\frac{\lambda+u_{y}\left(c\left(n^{k}\right), y\left(n^{k}\right), n^{k}\right)}{u_{y}\left(c\left(n^{k}\right), y\left(n^{k}\right), n^{k}\right)} \leq 0
$$

for all $k$. (5.22) and (5.23) imply that $u_{y}\left(c\left(n_{j}\right), y\left(n_{j}\right), n_{j}\right) \geq u_{y}\left(c\left(n^{k}\right), y\left(n^{k}\right), n^{k}\right)$ for all $j$ and $k$. 
However, for any sufficiently large $j$ and $k$, one also has $c\left(n_{j}\right)=c\left(n^{k}\right)$ and $y\left(n_{j}\right)>y\left(n^{k}\right)$. For such $j$ and $k$, RMC implies $u_{y}\left(c\left(n^{k}\right), y\left(n^{k}\right), n_{j}\right)>$ $u_{y}\left(c\left(n^{k}\right), y\left(n^{k}\right), n^{k}\right)$, contrary to part (a) of DR. The assumption that, for some $n, n^{\prime}>n$, and $\hat{n} \in\left(n, n^{\prime}\right)$, one has $c(n)=c\left(n^{\prime}\right)$ and $y(\cdot)$ strictly decreasing at $\hat{n}$ has thus led to a contradiction and must be false.

Lemma 5.3 The Lagrange multiplier of the feasibility constraint is strictly positive, i.e. $\lambda>0$.

In the finite-type case, the assertion of Lemma 5.3 is trivial because any slack in the feasibility constraint can be used to raise the highest type's consumption without upsetting downward incentive compatibility or consumption monotonicity. In the continuous-type case, the conclusion is still true, but one has to pay more attention to the constraints. The argument, which is given in the Appendix, is purely technical and provides no additional insight.

The next lemma shows that consumption and output provision are (almost) never inefficiently high. This corresponds to Lemma 4.2 in the finitetype case. A minor complication is caused by the possibility that $v(\cdot)$ or $c(\cdot)$ might be discontinuous. Relying on the monotonicity of $v(\cdot)$ and $c(\cdot)$, I therefore introduce the left-hand limits $\hat{v}(n)=\lim _{n^{\prime} \uparrow n} v\left(n^{\prime}\right), \hat{c}(n)=\lim _{n^{\prime} \uparrow n} c\left(n^{\prime}\right)$. By RMQ, one can also define $\hat{y}(n)$ so that $\hat{v}(n)=u(\hat{c}(n), \hat{y}(n), n)$, and one has $\hat{y}(n)=\lim _{n^{\prime} \uparrow n} y\left(n^{\prime}\right)$. Further, using abbreviated notation again, I write $\left(c^{*}(n), y^{*}(n)\right)$ and $\left(\hat{c}^{*}(n), \hat{y}^{*}(n)\right)$ for the efficient pairs $\left(c^{*}(n, v(n)), y^{*}(n, v(n))\right)$ and $\left(c^{*}(n, \hat{v}(n)), y^{*}(n, \hat{v}(n))\right)$ corresponding to the utility levels $v(n)$ and $\hat{v}(n)$.

Lemma 5.4 For all $n \in N,(\hat{c}(n), \hat{y}(n)) \leq\left(\hat{c}^{*}(n), \hat{y}^{*}(n)\right)$. In particular, $(c(n), y(n)) \leq\left(c^{*}(n), y^{*}(n)\right)$ for all $n \in N$ at which $(c(\cdot), y(\cdot))$ is continuous.

Proof. If the lemma is false, one has $(\hat{c}(\hat{n}), \hat{y}(\hat{n}))>>\left(\hat{c}^{*}(\hat{n}), \hat{y}^{*}(\hat{n})\right)$ for some $\hat{n} \in N$. Because $\hat{v}(\hat{n})=\lim _{n \uparrow \hat{n}} v(n)$, RMC implies that

$$
\left(\hat{c}^{*}(\hat{n}), \hat{y}^{*}(\hat{n})\right)=\lim _{n \uparrow \hat{n}}\left(c^{*}(n), y^{*}(n)\right) .
$$

By the definition of $(\hat{c}(\hat{n}), \hat{y}(\hat{n}))$, it follows that, for some $\delta>0$, one has $(c(n), y(n))>>\left(c^{*}(n), y^{*}(n)\right)$ for all $n \in(\hat{n}-\delta, \hat{n})$. It follows that $c(n)>0$ 
and $y(n)>0$ for all $n \in(\hat{n}-\delta, \hat{n})$. On the interval $(\hat{n}-\delta, \hat{n}),(5.17)$ must therefore hold as an equation, i.e., one has

$$
\psi^{\prime}(n)=\lambda \frac{u_{c}+u_{y}}{u_{y}} f(n)-\varphi(n)\left(u_{n c}-\frac{u_{c}}{u_{y}} u_{n y}\right) .
$$

By RMQ, the inequality $(c(n), y(n))>>\left(c^{*}(n), y^{*}(n)\right)$, and Lemma 5.3, the first term on the right-hand side of (5.24) is strictly positive. By SSCC and (5.8), the second term on the right-hand side of (5.24) is nonnegative. Therefore, $\psi^{\prime}(n)>0$ for $n \in(\hat{n}-\delta, \hat{n})$. Since $(5.9)$ implies $\psi(\hat{n}) \leq 0$, it follows that $\psi(n)<0$ for all $n \in(\hat{n}-\delta, \hat{n})$.

By the transversality condition $\psi\left(n^{0}\right)=0$, the set $\{n<\hat{n}-\delta \mid \psi(n)=0\}$ is nonempty. Let $\bar{n}$ be the supremum of this set. By the continuity of $\psi$, one has $\psi(\bar{n})=0$ and $\bar{n}<\hat{n}-\delta$. By the definition of $\bar{n}$, one also has $\psi(n)<0$ for all $n \in(\bar{n}, \hat{n})$. Therefore, $c(n)=c(\hat{n})$ for all $n \in(\bar{n}, \hat{n})$. By Lemma 5.2, it follows that $y(n)=y(\hat{n})$ for all $n \in(\bar{n}, \hat{n}+\delta)$. However, by SSCC, the inequality

$$
u_{c}(c(\hat{n}), y(\hat{n}), \hat{n})+u_{y}(c(\hat{n}), y(\hat{n}), \hat{n})<0
$$

implies that

$$
u_{c}(c(\hat{n}), y(\hat{n}), n)+u_{y}(c(\hat{n}), y(\hat{n}), n)<0
$$

for all $n<\hat{n}$. By (5.24), it follows that $\psi^{\prime}(n)>0$ for $n \in(\bar{n}, \hat{n}-\delta)$, as well as $n \in(\hat{n}-\delta, \hat{n})$. Since $\psi(\hat{n}-\delta)<0$, it follows that $\psi(\bar{n})<0$, contrary to the definition of $\bar{n}$. The assumption that $(\hat{c}(\hat{n}), \hat{y}(\hat{n}))>>\left(\hat{c}^{*}(\hat{n}), \hat{y}^{*}(\hat{n})\right)$ for some $\hat{n} \in N$ thus leads to a contradiction and must be false.

The next lemma shows that, at any $n \in\left(n^{0}, n^{1}\right)$, either the monotonicity constraint on $S(\cdot)$ or the monotonicity constraint on consumption must be strictly binding. This corresponds to Lemma 4.3 in the finite-type case. Again, part (b) of condition DR is central to the argument.

Lemma 5.5 For any $n \in\left(n^{0}, n^{1}\right)$, at least one of the costate variables $\varphi(n)$, $\psi(n)$ is nonzero.

The proof of Lemma 5.5 follows the same line of argument as the proof of Lemma 4.3, namely:

Step 1: If $\varphi(\hat{n})=\psi(\hat{n})=0$ for some $\hat{n} \in\left(n^{0}, n^{1}\right)$, then $(\hat{c}(\hat{n}), \hat{y}(\hat{n}))=$ $\left(\hat{c}^{*}(n), \hat{y}^{*}(n)\right)$, i.e., the left-hand $\operatorname{limit}_{n} \lim _{n \uparrow \hat{n}}(c(n), y(n))$ is efficient for $\hat{n}$. $\lambda$.

Step 2: If $\varphi(\hat{n})=\psi(\hat{n})=0$ for some $\hat{n} \in\left(n^{0}, n^{1}\right)$, then $\left|u_{y}(\hat{c}(\hat{n}), \hat{y}(\hat{n}), \hat{n})\right| \leq$ 
Step 3: If $\varphi(\hat{n})=\psi(\hat{n})=0$ for some $\hat{n} \in\left(n^{0}, n^{1}\right)$, then there exists a sequence $\left\{n^{k}\right\}$, which converges to $\hat{n}$ from above, such that $\left|u_{y}\left(c\left(n^{k}\right), y\left(n^{k}\right), n^{k}\right)\right| \geq$ $\lambda$ for all $k$.

Step 4: The conclusions of Steps 1 - 3 are in conflict with part (b) of condition DR.

However, in the continuous-type case, the argument for each of these steps is significantly more involved than in the finite-type case. The first three steps are therefore given as separate lemmas.

Lemma 5.6 For any $\hat{n} \in\left(n^{0}, n^{1}\right], \varphi(\hat{n})=\psi(\hat{n})=0$ implies

$$
(\hat{c}(\hat{n}), \hat{y}(\hat{n}))=\left(\hat{c}^{*}(n), \hat{y}^{*}(n)\right) .
$$

Moreover, if $\hat{c}(\hat{n})>0$, then $c(n)<\hat{c}(\hat{n})$ for $n<\hat{n}$.

Proof. $\psi(\hat{n})=0$ implies that, for some sequence $\left\{n^{k}\right\}$ which converges to $\hat{n}$ from below, one has $\psi^{\prime}\left(n^{k}\right) \geq 0$ for all $k$. For any element of this sequence, (5.18) yields

$$
\varphi\left(n^{k}\right)\left(u_{n c}^{k}-\frac{u_{c}^{k}}{u_{y}^{k}} u_{n y}^{k}\right) \leq \lambda \frac{u_{c}^{k}+u_{y}^{k}}{u_{y}^{k}} f\left(n^{k}\right) .
$$

By the continuity of $\varphi, \varphi(\hat{n})=0$ implies that the left-hand side of (5.27) goes to zero as $n^{k}$ converges to $\hat{n}$. Since $u_{y}^{k}<0$ for all $k$, it follows that

$$
\lim _{k \rightarrow \infty}\left(u_{c}^{k}+u_{y}^{k}\right) \leq 0,
$$

and hence, by RMQ, that

$$
u_{c}(\hat{c}(\hat{n}), \hat{y}(\hat{n}), \hat{n})+u_{y}(\hat{c}(\hat{n}), \hat{y}(\hat{n}), \hat{n}) \leq 0 .
$$

Thus $(\hat{c}(\hat{n}), \hat{y}(\hat{n})) \geq\left(\hat{c}^{*}(\hat{n}), \hat{y}^{*}(\hat{n})\right)$. By Lemma 5.4 and RMQ, one also has $(\hat{c}(\hat{n}), \hat{y}(\hat{n})) \leq\left(\hat{c}^{*}(\hat{n}), \hat{y}^{*}(\hat{n})\right)$. (5.26) follows immediately.

By PEP, it follows that $\hat{y}^{*}(\hat{n})>0$. If $\hat{c}(\hat{n})>0$, the first-order condition for $\left(\hat{c}^{*}(\hat{n}), \hat{y}^{*}(\hat{n})\right)$ implies that

$$
u_{c}(\hat{c}(\hat{n}), \hat{y}(\hat{n}), \hat{n})+u_{y}(\hat{c}(\hat{n}), \hat{y}(\hat{n}), \hat{n})=0 .
$$

For $n<\hat{n}$ therefore, SSCC implies

$$
u_{c}(\hat{c}(\hat{n}), \hat{y}(\hat{n}), n)+u_{y}(\hat{c}(\hat{n}), \hat{y}(\hat{n}), n)<0,
$$

so Lemma 5.4 yields $(c(n), y(n)) \neq(\hat{c}(\hat{n}), \hat{y}(\hat{n}))$. By Lemma 5.2, it follows that $c(n) \neq \hat{c}(\hat{n})$. By consumption monotonicity, therefore, $c(n)<\hat{c}(\hat{n})$ for $n<\hat{n}$. 
Lemma 5.7 For any $\hat{n} \in\left(n^{0}, n^{1}\right), \varphi(\hat{n})=\psi(\hat{n})=0$ implies

$$
\left|u_{y}(\hat{c}(\hat{n}), \hat{y}(\hat{n}), \hat{n})\right| \leq \lambda \text {. }
$$

Proof. Let $\hat{n} \in\left(n^{0}, n^{1}\right)$ be such that $\varphi(\hat{n})=\psi(\hat{n})=0$. I distinguish two cases according to whether $\hat{c}(\hat{n})$ is zero or positive. In either case, Lemma 5.6 yields (5.26), so PEP implies $\hat{y}(\hat{n})>0$.

If $\hat{c}(\hat{n})=0$, then, by the monotonicity of consumption and Lemma 5.2 , one must have $c(n)=0$ and $y(n)=\hat{y}(\hat{n})$ for $n<\hat{n}$. Positivity of $\hat{y}(\hat{n})$ implies that, for $n \in\left(n^{0}, \hat{n}\right),(5.16)$ must hold as an equation. With $c(n)=0$ and $y(n)=\hat{y}(\hat{n})$, this equation takes the form

$$
\lambda f(n)+u_{y}(0, \hat{y}(\hat{n}), n) f(n)+\frac{d}{d n} \varphi(n) u_{y}(0, \hat{y}(\hat{n}), n)=0 .
$$

Now RMC and (5.8) imply that $\varphi(n) u_{y}(0, \hat{y}(\hat{n}), n) \geq 0$ for all $n$. Because $\varphi(\hat{n}) u_{y}(0, \hat{y}(\hat{n}), \hat{n})=0$, it follows that, for some sequence $\left\{n_{j}\right\}$ which converges to $\hat{n}$ from below, one has $\frac{d}{d n}\left[\varphi(\cdot) u_{y}(0, \hat{y}(\hat{n}), \cdot)\right]\left(n_{j}\right) \leq 0$ for all $j$, hence $\lambda f\left(n_{j}\right)+u_{y}\left(0, \hat{y}(\hat{n}), n_{j}\right) f\left(n_{j}\right) \geq 0$ for all $j$. Upon taking limits as $n_{j}$ converges to $\hat{n}$, one finds that $\left|u_{y}(0, \hat{y}(\hat{n}), \hat{n})\right| \leq \lambda$, which is just (5.30).

If $\hat{c}(\hat{n})>0$, then, by Lemma 5.6, one has $\hat{c}(\hat{n})>c(n)$ for all $n<\hat{n}$. Therefore there exists a sequence $\left\{n^{k}\right\}$ converging to $\hat{n}$ from below such that $\psi\left(n^{k}\right)=0$ for all $k$. Because $\hat{c}(\hat{n})>0$, one may also suppose that, for some $k_{0}$, (5.15) must hold as an equation for all $n \geq n^{k_{0}}$. This equation can be rewritten as:

$$
\frac{d}{d n}\left(\varphi(n) e^{\int_{n}^{n} \frac{u_{n c}}{u_{c}} d n^{\prime}}\right)=\left[\frac{\lambda-u_{c}}{u_{c}} f(n)-\frac{\psi^{\prime}(n)}{u_{c}}\right] e^{\int_{n^{0}}^{n} \frac{u_{n c}}{u_{c}} d n^{\prime}},
$$

so integration between $n^{k}$ and $\hat{n}$ yields:

$$
\begin{aligned}
\varphi(\hat{n}) e^{\int_{n}^{\hat{n}} \frac{u_{n c}}{u_{c}} d n^{\prime}}= & \varphi\left(n^{k}\right) e^{\int_{n^{0}}^{n^{k}} \frac{u_{n c}}{u_{c}} d n^{\prime}}+\int_{n^{K}}^{\hat{n}} \frac{\lambda-u_{c}}{u_{c}} f(n) e^{\int_{n}^{n} \frac{u_{n c}}{u c} d n^{\prime}} d n \\
& -\int_{n^{K}}^{\hat{n}} \frac{\psi^{\prime}(n)}{u_{c}} f(n) e^{\int_{n^{0}}^{n} \frac{u_{n c}}{u_{c}} d n^{\prime}} d n .
\end{aligned}
$$

Because $\varphi(\hat{n})=0$ and, by (5.8), $\varphi\left(n^{k}\right) \leq 0$, it follows that

$$
\int_{n^{k}}^{\hat{n}} \frac{\lambda-u_{c}}{u_{c}} f(n) e^{\int_{n}^{n} \frac{u_{n c}}{u c} d n^{\prime}} d n \geq \int_{n^{k}}^{\hat{n}} \frac{\psi^{\prime}(n)}{u_{c}} f(n) e^{\int_{n^{0}}^{n} \frac{u_{n c}}{u c} d n^{\prime}} d n
$$

for all $k$. Lemma A.4 in the Appendix shows that the right-hand side of (5.34) is equal to zero for all $k$. Thus,

$$
\int_{n^{k}}^{\hat{n}} \frac{\lambda-u_{c}}{u_{c}} f(n) e^{\int_{n^{0}}^{n} \frac{u_{n c}}{u c} d n^{\prime}} d n \geq 0
$$


for all $k$. (5.35) holding, regardless of $k$, implies that, for some sequence $\left\{n_{j}\right\}$ converging to $\hat{n}$ from below, one has $\lambda \geq u_{c}\left(c\left(n_{j}\right), y\left(n_{j}\right), n_{j}\right)$ for all $j$. By RMQ, it follows that $\lambda \geq u_{c}(\hat{c}(\hat{n}), \hat{y}(\hat{n}), \hat{n})$. To establish (5.30), it now suffices to observe that, with $\hat{c}(\hat{n})>0$ and $\hat{y}(\hat{n})>0$ (5.26) implies

$$
u_{c}(\hat{c}(\hat{n}), \hat{y}(\hat{n}), \hat{n})+u_{y}(\hat{c}(\hat{n}), \hat{y}(\hat{n}), \hat{n})=0 .
$$

Lemma 5.8 For any $\hat{n} \in\left(n^{0}, n^{1}\right), \varphi(\hat{n})=\psi(\hat{n})=0$ implies that, for some sequence $\left\{n^{k}\right\}$ which converges to $\hat{n}$ from above, one has

$$
\left|u_{y}\left(c\left(n^{k}\right), y\left(n^{k}\right), n^{k}\right)\right| \geq \lambda
$$

for all $k$.

Proof. Let $\hat{n} \in\left(n^{0}, n^{1}\right)$ be such that $\varphi(\hat{n})=\psi(\hat{n})=0$. As in the proof of Lemma 5.2, let $h_{y}(n)=\varphi(n) \exp \left(\int_{n_{0}}^{n} \frac{u_{n y}}{u_{y}} d n^{\prime}\right)$, so (5.8) implies $h_{y}(n) \leq 0$ for all $n$, and $\varphi(\hat{n})=0$ implies $h_{y}(\hat{n})=0$. Because $h_{y}(\cdot)$ has a maximum at $\hat{n}$, there exists a sequence $\left\{n^{k}\right\}$ which converges to $\hat{n}$ from above, such that $h_{y}^{\prime}\left(n^{k}\right) \leq 0$ for all $k$, hence

$$
\varphi^{\prime}\left(n^{k}\right)+\varphi\left(n^{k}\right) \frac{u_{n y}\left(c\left(n^{k}\right), y\left(n^{k}\right), n^{k}\right)}{u_{y}\left(c\left(n^{k}\right), y\left(n^{k}\right), n^{k}\right)} \leq 0
$$

for all $k$. By (5.16), it follows that

$$
\frac{\lambda+u_{y}\left(c\left(n^{k}\right), y\left(n^{k}\right), n^{k}\right)}{u_{y}\left(c\left(n^{k}\right), y\left(n^{k}\right), n^{k}\right)} \geq 0
$$

for all $k$. Given that $u_{y}\left(c\left(n^{k}\right), y\left(n^{k}\right), n^{k}\right)<0,(5.36)$ follows immediately.

Proof of Lemma 5.5. By downward incentive compatibility, Lemma 5.6, and Lemma 5.4, the triples $(\hat{c}(\hat{n}), \hat{y}(\hat{n}), \hat{n})$ and $\left(c\left(n^{k}\right), y\left(n^{k}\right), n^{k}\right)$ satisfy all the premises of part (b) of DR. For any sufficiently large $k$, one therefore has $\left|u_{y}\left(c\left(n^{k}\right), y\left(n^{k}\right), n^{k}\right)\right|<\left|u_{y}(\hat{c}(\hat{n}), \hat{y}(\hat{n}), \hat{n})\right|$. However, this is incompatible with (5.30) and (5.36).

Given Lemma 5.5, the following lemma shows that downward incentive compatibility is everywhere locally binding. This is the analogue of Lemma 4.4 in the finite-type case. 
Lemma 5.9 For any $n \in\left(n^{0}, n^{1}\right)$,

$$
\frac{d v}{d n}(n)=u_{n}(c(n), y(n), n) .
$$

Proof. If the lemma is false, then, by (5.4), there exists a point $\hat{n} \in$ $\left(n^{0}, n^{1}\right)$ at which the slack function $S(\cdot)$ is strictly increasing. By the complementary slackness condition associated with (5.8), it follows that $\varphi(\hat{n})=0$. By Lemma 5.5, it follows that $\psi(\hat{n})<0$. Because $\psi$ is continuous, one must have $\psi(n)<0$ for all $n$ in some neighbourhood $[\hat{n}-\delta, \hat{n}+\delta]$ of $\hat{n}$. By the complementary slackness condition associated with (5.9), it follows that $(c(n), y(n))=(c(\hat{n}), y(\hat{n}))$ for all $n \in[\hat{n}-\delta, \hat{n}+\delta]$. Hence

$$
\begin{aligned}
v(\hat{n}+\delta)-v(\hat{n}-\delta) & =u(c(\hat{n}), y(\hat{n}), \hat{n}+\delta)-u(c(\hat{n}), y(\hat{n}), \hat{n}-\delta) \\
& =\int_{\hat{n}-\delta}^{\hat{n}+\delta} u_{n}(c(n), y(n), n) d n .
\end{aligned}
$$

By (5.4), it follows that $S(\hat{n}+\delta)-S(\hat{n}-\delta)=0$, contrary to the assumption that $S(\cdot)$ is increasing at $\hat{n}$. The assumption that $S(\cdot)$ is increasing at $\hat{n}$ must therefore be false.

From (5.37), RMQ, and SSCC, in combination with the continuity of the allocation at $n^{0}$ and $n^{1}$, one immediately obtains:

Corollary 5.10 The functions $c(\cdot)$ and $y(\cdot)$ are co-monotonic, i.e., $y(\cdot)$ is increasing at any point at which $c(\cdot)$ is increasing and constant on any set on which $c(\cdot)$ is constant. Moreover, the allocation $(c(\cdot), y(\cdot))$ is incentive compatible.

Relying on Lemmas 5.4 and 5.9, the following two lemmas replicate Lemmas 4.6 and 4.7, showing that the difference between consumption and required output is nondecreasing and that consumption must be positive.

Lemma 5.11 The function $n \rightarrow c(n)-y(n)$ is nonincreasing on $N$; on any subset of $N$ on which $(c(n), y(n))$ is distorted downward from efficiency, the function $n \rightarrow c(n)-y(n)$ is strictly decreasing.

Proof. For any $\hat{n} \in\left[n^{0}, n^{1}\right)$ and any $n \in\left(\hat{n}, n^{1}\right]$, one can write

$$
c(n)-y(n)=c(\hat{n})-y(\hat{n})+\int_{\hat{n}}^{n} d c\left(n^{\prime}\right)-\int_{\hat{n}}^{n} d y\left(n^{\prime}\right) .
$$


By (5.37), one has

$$
\int_{\hat{n}}^{n} d c\left(n^{\prime}\right)=-\int_{\hat{n}}^{n} \frac{u_{y}\left(c\left(n^{\prime}\right), y\left(n^{\prime}\right), n^{\prime}\right)}{u_{c}\left(c\left(n^{\prime}\right), y\left(n^{\prime}\right), n^{\prime}\right)} d y\left(n^{\prime}\right) .
$$

Because Lemma 5.4 implies that $u_{c}\left(c\left(n^{\prime}\right), y\left(n^{\prime}\right), n^{\prime}\right) \geq-u_{y}\left(c\left(n^{\prime}\right), y\left(n^{\prime}\right), n^{\prime}\right)$ for almost all $n^{\prime}$, with a strict inequality if $\left(c\left(n^{\prime}\right), y\left(n^{\prime}\right)\right)$ is distorted downward from efficiency, the result follows.

Lemma 5.12 For $n \in N$, consumption is bounded away from zero.

Proof. If the lemma is false, one has $c\left(n^{0}\right)=0$. By Lemma 5.11, one then has

$$
c(n)-y(n) \leq-y\left(n^{0}\right) \leq 0
$$

for all $n \in N$. But then, for all $n \in N$, one has

$$
u(c(n), y(n), n) \leq u\left(y^{L F}(n), y^{L F}(n), n\right),
$$

where, for any $n, y^{L F}(n)$ is the laissez-faire level of output provision and consumption, i.e.,

$$
y^{L F}(n)=\arg \max _{y} u(y, y, n) .
$$

The allocation $(c(\cdot), y(\cdot))$ would thus be dominated by the laissez-faire allocation $\left(y^{L F}(\cdot), y^{L F}(\cdot)\right)$ and could not be a canonical solution to the weakly relaxed income tax problem unless one had $(c(n), y(n))=\left(y^{L F}(n), y^{L F}(n)\right)$ for almost all $n \in N$. Given that $c\left(n^{0}\right)=0$, it follows that $y^{L F}\left(n^{0}\right)=0$. By $\mathrm{PEP}$, this is impossible.

Lemma 5.13 On any compact subset of $\left(n^{0}, n^{1}\right),(c(n), y(n))$ is distorted downward and bounded away from efficiency.

Proof. If the lemma is false, there exists a sequence $\left\{n_{j}\right\}$ converging to some $\hat{n} \in\left(n^{0}, n^{1}\right)$ such that

$$
\lim _{j \rightarrow \infty}\left(c\left(n_{j}\right), y\left(n_{j}\right)\right)=\lim _{j \rightarrow \infty}\left(c^{*}\left(n_{j}\right), y^{*}\left(n_{j}\right)\right) .
$$

By Lemma 5.9, the function $v(\cdot)$ is continuous at $\hat{n}$. Therefore,

$$
\lim _{k \rightarrow \infty}\left(c^{*}\left(n_{j}\right), y^{*}\left(n_{j}\right)\right)=\left(c^{*}(\hat{n}), y^{*}(\hat{n})\right) .
$$


By (5.43) and Lemma 5.12, one has $c^{*}(\hat{n})>0$ and, by PEP, $y^{*}(\hat{n})>0$. Therefore,

$$
u_{c}\left(c^{*}(\hat{n}), y^{*}(\hat{n}), \hat{n}\right)+u_{y}\left(c^{*}(\hat{n}), y^{*}(\hat{n}), \hat{n}\right)=0 .
$$

I claim that $\psi(\hat{n})=0$. If $\psi(\hat{n})<0$, then, by the continuity of $\psi$, there exists $\delta>0$ such that $\psi(n)<0$ for all $n \in(\hat{n}-\delta, \hat{n}+\delta)$. Then $c(n)=c^{*}(\hat{n})$ and, by Lemma 5.2, $y(n)=y^{*}(\hat{n})$ for all $n \in(\hat{n}-\delta, \hat{n}+\delta)$. By SSCC and (5.44), it follows that $(c(\hat{n}), y(\hat{n}))>>\left(c^{*}(\hat{n}), y^{*}(\hat{n})\right)$ for all $n \in(\hat{n}-\delta, \hat{n})$, contrary to Lemma 5.4. The assumption that $\psi(\hat{n})<0$ thus leads to a contradiction and must be false.

Since $\psi(\hat{n})=0$ and, by (5.9), $\psi(n) \leq 0$ for $n>\hat{n}$, there exists a sequence $\left\{n^{k}\right\}$ which converges to $\hat{n}$ from above such that $\psi^{\prime}\left(n^{k}\right) \leq 0$ for all $k$. By the monotonicity of the allocation, one has

$$
\left(c\left(n^{k}\right), y\left(n^{k}\right)\right) \geq \lim _{j \rightarrow \infty}\left(c\left(n_{j}\right), y\left(n_{j}\right)\right)>>(0,0)
$$

for all $k$, so, for $n=n^{k},(5.17)$ must hold as an equation. Thus, $\psi^{\prime}\left(n^{k}\right) \leq 0$ implies

$$
\lambda \frac{u_{c}^{k}+u_{y}^{k}}{u_{y}^{k}} f\left(n^{k}\right)-\varphi\left(n^{k}\right)\left(u_{n c}^{k}-\frac{u_{c}^{k}}{u_{y}^{k}} u_{n y}^{k}\right) \leq 0
$$

where the derivatives $u_{c}^{k}, u_{y}^{k}$, etc. are all evaluated at $\left(c\left(n^{k}\right), y\left(n^{k}\right), n^{k}\right)$. By Lemma 5.4, one also has $\left(c\left(n^{k}\right), y\left(n^{k}\right)\right) \leq\left(c^{*}\left(n^{k}\right), y^{*}\left(n^{k}\right)\right)$. Upon combining this inequality with (5.45), (5.42), and (5.43), one sees that

$$
\lim _{k \rightarrow \infty}\left(c\left(n^{k}\right), y\left(n^{k}\right)\right)=\left(c^{*}(\hat{n}), y^{*}(\hat{n})\right) .
$$

By (5.44) and RMQ, it follows that

$$
\lim _{k \rightarrow \infty} \lambda \frac{u_{c}^{k}+u_{y}^{k}}{u_{y}^{k}} f\left(n^{k}\right)=0 .
$$

By RMQ and the continuity of $\varphi,(5.46)$ and (5.47) yield

$$
-\varphi(\hat{n})\left(u_{n c}-\frac{u_{c}}{u_{y}} u_{n y}\right) \leq 0 .
$$

By SSCC, $\left(u_{n c}-\frac{u_{c}}{u_{y}} u_{n y}\right)$ is strictly positive, so (5.48) implies $\varphi(\hat{n}) \geq 0$. By (5.8), it follows that $\varphi(\hat{n})=0$.

However, the finding that $\varphi(\hat{n})=\psi(\hat{n})=0$ contradicts Lemma 5.5. The assumption that (5.42) holds for some sequence $\left\{n_{j}\right\}$ converging to some $\hat{n} \in\left(n^{0}, n^{1}\right)$ must therefore be false. 
To complete the proof of Theorem 3.1 for the continuum case, I consider the behaviour of the allocation near the top and the bottom. Given the canonicity assumption that $\left(c\left(n^{1}\right), y\left(n^{1}\right)\right)=\left(\hat{c}\left(n^{1}\right), \hat{y}\left(n^{1}\right)\right)$, Lemma 5.6 and the transversality conditions (5.12) and (5.13) immediately yield $\left(c\left(n^{1}\right), y\left(n^{1}\right)\right)=$ $\left(c^{*}\left(n^{1}\right), y^{*}\left(n^{1}\right)\right)$, as well as $c\left(n^{1}\right)>c\left(n^{1}\right)$ for $n<n_{1}$. At the bottom, matters are slightly more complicated.

Lemma 5.14 If $c(\cdot)$ and $y(\cdot)$ are strictly increasing at $n^{0}$, then

$$
\left(c\left(n^{0}\right), y\left(n^{0}\right)\right)=\left(c^{*}\left(n^{0}, v\left(n^{0}\right)\right), y^{*}\left(n^{0}, v\left(n^{0}\right)\right)\right) ;
$$

if the monotonicity constraint on $c(\cdot)$ is strictly binding at $n_{0}$, then

$$
\left(c\left(n^{0}\right), y\left(n^{0}\right)\right)<<\left(c^{*}\left(n^{0}, v\left(n^{0}\right)\right), y^{*}\left(n^{0}, v\left(n^{0}\right)\right)\right) .
$$

Proof. Let $\left\{n^{k}\right\}$ be any sequence which converges to $n^{0}$ from above. Because $c\left(n^{0}\right)>0$, one must have $c\left(n^{k}\right)>0$ and $y\left(n^{k}\right)>0$ for all $k$.

Then (5.17) becomes

$$
\psi^{\prime}\left(n^{k}\right)=\lambda \frac{u_{c}^{k}+u_{y}^{k}}{u_{y}^{k}} f\left(n^{k}\right)-\varphi\left(n^{k}\right)\left(u_{n c}^{k}-\frac{u_{c}^{k}}{u_{y}^{k}} u_{n y}^{k}\right)
$$

for all $k$. Because $\varphi$ and $f$, as well as the allocation $(c(\cdot), y(\cdot))$ are continuous at $n_{0}$, it follows that $\psi^{\prime}\left(n^{k}\right)$ converges to a limit $\psi^{\prime}\left(n_{0}\right)$. By the transversality condition (5.12), the term $\varphi\left(n^{k}\right)\left(u_{n c}^{k}-\frac{u_{c}^{k}}{u_{y}^{k}} u_{n y}^{k}\right)$ on the right-hand side of (5.51) goes to zero as $k$ becomes large. Therefore

$$
\psi^{\prime}\left(n^{0}\right)=\lambda \frac{u_{c}\left(c\left(n^{0}\right), y\left(n^{0}\right), n^{0}\right)+u_{y}\left(c\left(n^{0}\right), y\left(n^{0}\right), n^{0}\right)}{u_{y}\left(c\left(n^{0}\right), y\left(n^{0}\right), n^{0}\right)} f\left(n^{0}\right) .
$$

If $c(\cdot)$ and $y(\cdot)$ are strictly increasing at $n^{0}$, the sequence $\left\{n^{k}\right\}$ can be chosen in such a way that $\psi^{\prime}\left(n^{k}\right) \geq 0$ for all $k$. Given that $\psi\left(n^{0}\right)=0$ and $\psi\left(n^{k}\right) \leq 0$ for all $k$, it follows that $\psi^{\prime}\left(n^{0}\right)=0$, so (5.52) implies (5.49). In contrast, if $\psi^{\prime}\left(n^{0}\right)<0,(5.52)$ implies (5.50).

The proof of Theorem 3.1 for the continuous-type case is now complete. Theorems 3.2 and 3.3 follow from Theorem 3.1 by the argument given in the text. 


\section{A Appendix: Supplementary Proofs}

\section{A.1 Proof of Proposition 2.2}

The proof of Proposition 2.2 proceeds in several steps. Each step corresponds to a different subset of the set of pairs $\left(c^{\prime}, y^{\prime}\right)$ for which the inequality $(2.13)$ is deemed to hold. For a better understanding of the structure, it will be useful to refer back to Figure 1.

The first step concerns those outcome pairs $\left(c^{\prime}, y^{\prime}\right)$ that lie on the horizontal line from the pair which is assigned to type $n$ to the vertical axis. The following claim shows that, for such outcome pairs, the inequality (2.13) holds regardless of whether the pair which is assigned to type $n$ is efficient or not. This claim follows directly from the concavity of $u$ and (2.15).

Claim A.1 If $(c, y, n) \in \Re_{+}^{2} \times\left[n^{0}, n^{1}\right)$ and $\left(c^{\prime}, y^{\prime}, n^{\prime}\right) \in \Re_{+}^{2} \times\left(n, n_{1}\right]$ are such that $c^{\prime}=c$ and $y^{\prime} \leq y$, then $\left|u_{y}\left(c^{\prime}, y^{\prime}, n^{\prime}\right)\right|<\left|u_{y}(c, y, n)\right|$.

The second step concerns outcome pairs $\left(c^{\prime}, y^{\prime}\right)$ that lie on the indifference curve $I\left(n^{\prime}\right)$ of type $n^{\prime}$ between the reference point $\left(c^{*}(n), y^{*}(n)\right)$ and the point $\left(c^{*}\left(n^{\prime}\right), y^{*}\left(n^{\prime}\right)\right)$ that is efficient for $n^{\prime}$.

Claim A.2 For any $(c, y, n) \in \Re_{+}^{2} \times\left[n^{0}, n^{1}\right)$ such that $(c, y)$ is efficient for $n$, there exists $\varepsilon>0$ such that $\left|u_{y}\left(c^{\prime}, y^{\prime}, n^{\prime}\right)\right|<\left|u_{y}(c, y, n)\right|$ for any $n^{\prime} \in$ $(n, n+\varepsilon]$ and any $\left(c^{\prime}, y^{\prime}\right)$ that satisfies

$$
u\left(c^{\prime}, y^{\prime}, n^{\prime}\right)=u\left(c, y, n^{\prime}\right),
$$

as well as $c \leq c^{\prime} \leq c^{*}\left(n^{\prime}, u\left(c, y, n^{\prime}\right)\right)$.

Proof. If $c=0$ or $y=0$ and if the first-order condition for $(c, y)$ to be efficient for $n$,

$$
u_{c}(c, y, n)+u_{y}(c, y, n) \leq 0,
$$

holds as a strict inequality, then, for $n^{\prime}$ sufficiently close to $n,(c, y)$ must also be efficient for $n^{\prime}$. In this case, (A.1) and $c \leq c^{\prime} \leq c^{*}\left(n^{\prime}, u\left(c, y, n^{\prime}\right)\right)$ imply $\left(c^{\prime}, y^{\prime}\right)=(c, y)$, hence

$$
u_{y}\left(c^{\prime}, y^{\prime}, n^{\prime}\right)-u_{y}(c, y, n)=\int_{n}^{n^{\prime}} u_{y n}\left(c, y, n^{\prime \prime}\right) d n^{\prime \prime},
$$

and the claim follows from (2.15).

Alternatively, if $(c, y)$ satisfies the equation

$$
u_{c}(c, y, n)+u_{y}(c, y, n)=0,
$$


then, for $n^{\prime}>n$, SSCC implies

$$
u_{c}\left(c, y, n^{\prime}\right)+u_{y}\left(c, y, n^{\prime}\right)>0
$$

By PEP, there exists a unique pair $\left(c^{*}\left(n^{\prime}, u\left(c, y, n^{\prime}\right)\right), y^{*}\left(n^{\prime}, u\left(c, y, n^{\prime}\right)\right)\right)$ that lies on $I\left(n^{\prime}\right)$ and is efficient for $n^{\prime}$. This pair is strictly greater than $(c, y)$ and satisfies the first-order condition for an interior efficient point, $u_{c}+u_{y}=0$.

By RMQ, the indifference curve $I\left(n^{\prime}\right)$ is strictly convex. Between the reference point $(c, y)$ and the efficient pair for $n^{\prime}$, its slope is strictly decreasing from $\frac{u_{c}\left(c, y, n^{\prime}\right)}{\left|u_{y}\left(c, y, n^{\prime}\right)\right|}$ to one. For any point $\left(c^{\prime}, y^{\prime}\right)$ on $I\left(n^{\prime}\right)$ that satisfies $c \leq c^{\prime} \leq c^{*}\left(n^{\prime}, u\left(c, y, n^{\prime}\right)\right)$, there must therefore exist some $\delta \in[0,1]$ such that

$$
\frac{u_{c}\left(c^{\prime}, y^{\prime}, n^{\prime}\right)}{\left|u_{y}\left(c^{\prime}, y^{\prime}, n^{\prime}\right)\right|}=\delta \frac{u_{c}\left(c, y, n^{\prime}\right)}{\left|u_{y}\left(c, y, n^{\prime}\right)\right|}+(1-\delta) .
$$

Conversely, for the given $n^{\prime}$ and any $\delta \in[0,1]$, there exists a pair $\left(\hat{c}\left(n^{\prime}, \delta\right), \hat{y}\left(n^{\prime}, \delta\right)\right)$ such that $\left(c^{\prime}, y^{\prime}\right)=\left(\hat{c}\left(n^{\prime}, \delta\right), \hat{y}\left(n^{\prime}, \delta\right)\right)$ lies between $(c, y)$ and $\left(c^{*}\left(n^{\prime}\right), y^{*}\left(n^{\prime}\right)\right)$ and is the unique solution to equations (A.1) and (A.4). By construction, one has $(\hat{c}(n, \delta), \hat{y}(n, \delta))=(c, y)$ for all $\delta$. One also has $\left(\hat{c}\left(n^{\prime}, 0\right), \hat{y}\left(n^{\prime}, 0\right)\right)=$ $\left(c^{*}\left(n^{\prime}, u\left(c, y, n^{\prime}\right)\right), y^{*}\left(n^{\prime}, u\left(c, y, n^{\prime}\right)\right)\right)$ for all $n^{\prime}$.

By standard arguments, relying on the implicit function theorem, the assumption that indifference curves have positive Gaussian curvature implies that the functions $\hat{c}(\cdot, \cdot)$ and $\hat{y}(\cdot, \cdot)$ that are defined by (A.1) and (A.4) are continuously differentiable. It follows that, for any $\delta \in[0,1]$ and any $n^{\prime} \geq n$, the derivative

$$
\frac{d u_{y}\left(\hat{c}\left(n^{\prime}, \delta\right), \hat{y}\left(n^{\prime}, \delta\right), n^{\prime}\right)}{d n^{\prime}}=\left[u_{y c} \frac{\partial \hat{c}}{\partial n^{\prime}}\left(n^{\prime}, \delta\right)+u_{y y} \frac{\partial \hat{y}}{\partial n^{\prime}}\left(n^{\prime}, \delta\right)+u_{y n}\right]
$$

is well defined. At $n^{\prime}=n$ and any $\delta$, one computes ${ }^{22}$

$$
\frac{\partial \hat{c}}{\partial n^{\prime}}(n, \delta)=\frac{\partial \hat{y}}{\partial n^{\prime}}(n, \delta)=-(1-\delta) \frac{\left(u_{c n}+u_{y n}\right)}{u_{c c}+u_{c y}+u_{y c}+u_{y y}} .
$$

\footnotetext{
${ }^{22}$ Positivity of the Gaussian curvature implies that the denominator in (A.6) is strictly negative.
} 
For any $\delta \in[0,1]$, one therefore obtains

$$
\begin{aligned}
& \frac{d u_{y}\left(\hat{c}\left(n^{\prime}, \delta\right), \hat{y}\left(n^{\prime}, \delta\right), n^{\prime}\right)}{d n^{\prime}}(n, \delta) \\
= & -(1-\delta) \frac{\left(u_{c n}+u_{y n}\right)}{u_{c c}+u_{c y}+u_{y c}+u_{y y}}\left(u_{y c}+u_{y y}\right)+u_{y n} \\
= & (1-\delta)\left[-\frac{\left(u_{y c}+u_{y y}\right)}{u_{c c}+u_{c y}+u_{y c}+u_{y y}} u_{c n}+\frac{\left(u_{c c}+u_{c y}\right)}{u_{c c}+u_{c y}+u_{y c}+u_{y y}} u_{y n}\right]+\delta u_{y n} \\
= & (1-\delta)\left[u_{c n} \frac{\partial c^{*}(n, v)}{\partial v}+u_{y n} \frac{\partial y^{*}(n, v)}{\partial v}\right]+\delta u_{y n} \\
\geq & \min \left[-\left(u_{c n} \frac{\partial c^{*}(n, v)}{\partial v}+u_{y n} \frac{\partial y^{*}(n, v)}{\partial v}(n)\right), u_{y n}\right]
\end{aligned}
$$

The right-hand side of (A.7) is independent of $\delta$. By (2.15) and (2.16), it is also strictly positive. It follows that, for some $\varepsilon>0, n^{\prime} \in(n, n+\varepsilon]$ implies

$$
\frac{d u_{y}\left(\hat{c}\left(n^{\prime}, \delta\right), \hat{y}\left(n^{\prime}, \delta\right), n^{\prime}\right)}{d n^{\prime}}\left(n^{\prime}, \delta\right)>0
$$

for all $\delta \in[0,1]$. The claim follows immediately.

The third step concerns those outcome pairs $\left(c^{\prime}, y^{\prime}\right)$ for type $n^{\prime}$ that lie in the shaded area to the left of the curve $A-A$ of efficient points in Figure 1 and strictly above the indifference curve $I\left(n^{\prime}\right)$, as well as the horizontal line through the reference point $\left(c^{*}(n), y^{*}(n)\right)$. The argument is adapted from Brunner (1995).

Claim A.3 Suppose that $(c, y)$ is efficient for $n$, and let $\varepsilon$ be given by Claim 2. Then $\left|u_{y}\left(c^{\prime}, y^{\prime}, n^{\prime}\right)\right|<\left|u_{y}(c, y, n)\right|$ for any $n^{\prime} \in(n, n+\varepsilon]$ and any $\left(c^{\prime}, y^{\prime}\right)$ such that $c^{\prime}>c, u\left(c^{\prime}, y^{\prime}, n^{\prime}\right)>u\left(c, y, n^{\prime}\right)$, and $u_{c}\left(c^{\prime}, y^{\prime}, n^{\prime}\right)+$ $u_{y}\left(c^{\prime}, y^{\prime}, n^{\prime}\right) \geq 0$.

Proof. Let $q$ be such that

$$
q u_{c}\left(c^{\prime}, y^{\prime}, n^{\prime}\right)+u_{y}\left(c^{\prime}, y^{\prime}, n^{\prime}\right)=0 .
$$

Because $u_{y}\left(c^{\prime}, y^{\prime}, n^{\prime}\right)<0$ and $u_{c}\left(c^{\prime}, y^{\prime}, n^{\prime}\right)+u_{y}\left(c^{\prime}, y^{\prime}, n^{\prime}\right) \geq 0$, one must have $q \in(0,1]$. For any $v$ in the range of $u$, let $(\bar{c}(v), \bar{y}(v))$ be the solution to the problem of minimizing $c^{\prime \prime}-q y^{\prime \prime}$ under the constraint that $u\left(c^{\prime \prime}, y^{\prime \prime}, n^{\prime}\right) \geq v$. The existence of such a solution follows from RMQ, PEP and the fact that 
$q \leq 1$. By RMQ, the solution satisfies $(\bar{c}(v), \bar{y}(v)) \leq\left(c^{*}\left(n^{\prime}, v\right), y^{*}\left(n^{\prime}, v\right)\right)$ for all $v$.

From (A.8), one easily finds that $\left(c^{\prime}, y^{\prime}\right)=\left(\bar{c}\left(u\left(c^{\prime}, y^{\prime}, n^{\prime}\right)\right), \bar{y}\left(u\left(c^{\prime}, y^{\prime}, n^{\prime}\right)\right)\right)$. Because $u$ is concave, one also has

$$
\left|u_{y}\left(\bar{c}(v), \bar{y}(v), n^{\prime}\right)\right| \geq\left|u_{y}\left(c^{\prime}, y^{\prime}, n^{\prime}\right)\right|
$$

whenever $v<u\left(c^{\prime}, y^{\prime}, n^{\prime}\right)$. In particular,

$$
\left|u_{y}\left(\bar{c}\left(u\left(c, y, n^{\prime}\right)\right), \bar{y}\left(u\left(c, y, n^{\prime}\right)\right), n^{\prime}\right)\right| \geq\left|u_{y}\left(c^{\prime}, y^{\prime}, n^{\prime}\right)\right| \text {. }
$$

If $\bar{c}\left(u\left(c, y, n^{\prime}\right)\right) \geq c$, the claim follows from (A.9) because Claim A.2 yields $\left|u_{y}\left(\bar{c}\left(u\left(c, y, n^{\prime}\right)\right), \bar{y}\left(u\left(c, y, n^{\prime}\right)\right), n^{\prime}\right)\right|<\left|u_{y}(c, y, n)\right|$. If $\bar{c}\left(u\left(c, y, n^{\prime}\right)\right)<c$, then, by the intermediate value theorem, there exists $\hat{v} \in\left(u\left(c, y, n^{\prime}\right), u\left(c^{\prime}, y^{\prime}, n^{\prime}\right)\right)$ such that $\bar{c}(\hat{v})=c$ and $\bar{y}(v)<y$. For this $\hat{v}$, Claim A.1 yields $\left|u_{y}\left(\bar{c}(\hat{v}), \bar{y}(\hat{v}), n^{\prime}\right)\right|<$ $\left|u_{y}(c, y, n)\right|$, so again, the claim follows from (A.9).

To complete the proof of Proposition 2.2, it suffices to note that, if $N$ is an interval, then there is no loss of generality in postulating that $n+\varepsilon$ is an element of $N$. The same is true if $N$ is a finite set and the elements of $N$ are sufficiently close.

\section{A.2 Additional Proofs for Section 5}

Proof of Lemma 5.1. The argument follows Mirrlees (1976). For any $n$, let $S(n)$ be the difference between $v(n)$ and the integral $\int_{n^{0}}^{n} u_{n}\left(c\left(n^{\prime}\right), y\left(n^{\prime}\right), n^{\prime}\right) d n^{\prime}$. If $S(\cdot)$ is a nondecreasing function, one has

$$
\int_{n^{\prime}}^{n} \chi\left(n^{\prime \prime}, n\right) d S(n) \geq 0
$$

for all $n$, all $n^{\prime}<n$, and every nonnegative-valued function $\chi$. By the definition of $S(\cdot)$, (A.10) is equivalent to the inequality

$$
\int_{n^{\prime}}^{n} \chi\left(n^{\prime \prime}, n\right)\left[u_{c}\left(c\left(n^{\prime \prime}\right), y\left(n^{\prime \prime}\right), n^{\prime \prime}\right) d c\left(n^{\prime \prime}\right)+u_{y}\left(c\left(n^{\prime \prime}\right), y\left(n^{\prime \prime}\right), n^{\prime \prime}\right) d y\left(n^{\prime \prime}\right)\right] \geq 0 .
$$

With $\chi\left(n^{\prime \prime}, n\right)=\frac{u_{y}\left(c\left(n^{\prime \prime}\right), y\left(n^{\prime \prime}\right), n\right)}{u_{y}\left(c\left(n^{\prime \prime}\right), y\left(n^{\prime \prime}\right), n^{\prime \prime}\right)}$, it follows that

$$
\begin{gathered}
\int_{n^{\prime}}^{n} u_{y}\left(c\left(n^{\prime \prime}\right), y\left(n^{\prime \prime}\right), n\right) d y\left(n^{\prime \prime}\right) \\
+\int_{n^{\prime}}^{n} \frac{u_{y}\left(c\left(n^{\prime \prime}\right), y\left(n^{\prime \prime}\right), n\right)}{u_{y}\left(c\left(n^{\prime \prime}\right), y\left(n^{\prime \prime}\right), n^{\prime \prime}\right)} u_{c}\left(c\left(n^{\prime \prime}\right), y\left(n^{\prime \prime}\right), n^{\prime \prime}\right) d c\left(n^{\prime \prime}\right) \geq 0
\end{gathered}
$$


for all $n$ and all $n^{\prime}<n$.

By SSCC and RMC, one also has

$$
\frac{-u_{y}\left(c\left(n^{\prime \prime}\right), y\left(n^{\prime \prime}\right), n\right)}{u_{c}\left(c\left(n^{\prime \prime}\right), y\left(n^{\prime \prime}\right), n\right)}<\frac{-u_{y}\left(c\left(n^{\prime \prime}\right), y\left(n^{\prime \prime}\right), n^{\prime \prime}\right)}{u_{c}\left(c\left(n^{\prime \prime}\right), y\left(n^{\prime \prime}\right), n^{\prime \prime}\right)},
$$

whenever $n^{\prime \prime}<n$. Because $c(\cdot)$ is nondecreasing, it follows that

$$
\begin{gathered}
-\int_{n^{\prime}}^{n} \frac{u_{y}\left(c\left(n^{\prime \prime}\right), y\left(n^{\prime \prime}\right), n\right)}{u_{y}\left(c\left(n^{\prime \prime}\right), y\left(n^{\prime \prime}\right), n^{\prime \prime}\right)} u_{c}\left(c\left(n^{\prime \prime}\right), y\left(n^{\prime \prime}\right), n^{\prime \prime}\right) d c\left(n^{\prime \prime}\right) \\
\geq-\int_{n^{\prime}}^{n} u_{c}\left(c\left(n^{\prime \prime}\right), y\left(n^{\prime \prime}\right), n\right) d c\left(n^{\prime \prime}\right)
\end{gathered}
$$

for all $n$ and all $n^{\prime}<n$. Now (A.11) and (A.12) imply

$$
\int_{n^{\prime}}^{n} u_{c}\left(c\left(n^{\prime \prime}\right), y\left(n^{\prime \prime}\right), n\right) d c\left(n^{\prime \prime}\right)+\int_{n^{\prime}}^{n} u_{y}\left(c\left(n^{\prime \prime}\right), y\left(n^{\prime \prime}\right), n\right) d y\left(n^{\prime \prime}\right) \geq 0
$$

hence

$$
u(c(n), y(n), n) \geq u\left(c\left(n^{\prime}\right), y\left(n^{\prime}\right), n\right)
$$

for all $n$ and all $n^{\prime}<n$. Monotonicity of $c(\cdot)$ and $S(\cdot)$ is thus sufficient for downward incentive compatibility.

Conversely, for all $n$ and all $n^{\prime}<n$, downward incentive compatibility implies

$$
v(n)-v\left(n^{\prime}\right) \geq \int_{n^{\prime}}^{n} u_{n}\left(c\left(n^{\prime}\right), y\left(n^{\prime}\right), n^{\prime \prime}\right) d n^{\prime \prime} .
$$

By standard arguments, it follows that

$$
\begin{aligned}
v(n)-v\left(n^{\prime}\right) & =\sum_{k=1}^{K}\left(v\left(n_{k+1}\right)-v\left(n_{k}\right)\right) \\
& \geq \sum_{k=1}^{K} \int_{n_{k}}^{n_{k+1}} u_{n}\left(c\left(n_{k}\right), y\left(n_{k}\right), n^{\prime \prime}\right) d n^{\prime \prime}
\end{aligned}
$$

for every increasing sequence $\left\{n_{k}\right\}_{k=1}^{K}$ with $n_{1}=n^{\prime}$ and $n_{K}=n$. Upon taking limits across sequences $\left\{n_{k}\right\}_{k=1}^{K}$ as $K$ goes out of bounds and the sequence $\left\{n_{k}\right\}_{k=1}^{K}$ becomes dense in the interval $\left[n^{\prime}, n\right]$, one concludes that

$$
v(n)-v\left(n^{\prime}\right) \geq \int_{n^{\prime}}^{n} u_{n}\left(c\left(n^{\prime \prime}\right), y\left(n^{\prime \prime}\right), n^{\prime \prime}\right) d n^{\prime \prime}
$$

and, hence, that $S(n) \geq S\left(n^{\prime}\right)$ for all $n$ and all $n^{\prime}<n$. 
Lemma A.4 For any $\hat{n}$ and $\bar{n} \in\left[n^{0}, n^{1}\right]$ such that $\psi(\hat{n})=0$ and $\psi(\bar{n}) \leq 0$, one has

$$
\int_{\bar{n}}^{\hat{n}} \frac{\psi^{\prime}(n)}{u_{c}} f(n) e^{\int_{n 0}^{n} \frac{u_{n c}}{u c} d n^{\prime}} d n \geq 0
$$

(A.17) holds with equality if and only if $\psi(\bar{n})=0$.

Proof. Without loss of generality, let $\bar{n}<\hat{n}$. Neglecting null sets, one may suppose that $\psi^{\prime}(n)$ is nonzero only on intervals of constancy of $c(\cdot)$. On such intervals, by Lemma $5.2, y(\cdot)$ is also constant. Let $\mathcal{I}$ be the collection of such intervals between $\bar{n}$ and $\hat{n}$ and note that $\mathcal{I}$ is at most countable. For any $I \in \mathcal{I}$, let $n_{0}(I)$ and $n_{1}(I)$ be the infimum and the supremum of $I$, and let $c(I), y(I)$ be the common value of $\left(c\left(n^{\prime}\right), y\left(n^{\prime}\right)\right)$ on $\left(n_{0}(I), n_{1}(I)\right)$. Because $\psi^{\prime}(n)$ may be taken to vanish outside the union of intervals in $\mathcal{I}$, one can write:

$$
\int_{\bar{n}}^{\hat{n}} \frac{\psi^{\prime}(n)}{u_{c}} f(n) e^{\int_{n^{0}}^{n} \frac{u_{n c}}{u_{c}} d n^{\prime}} d n=\sum_{I \in \mathcal{I}} \int_{n_{0}(I)}^{n_{1}(I)} \frac{\psi^{\prime}(n)}{u_{c}} e^{\int_{n^{0}}^{n} \frac{u_{n c}}{u_{c}} d n^{\prime}} d n .
$$

For any $I \in \mathcal{I}$ and any $n^{\prime} \in\left(n_{0}(I), n_{1}(I)\right)$, one has $\left(c\left(n^{\prime}\right), y\left(n^{\prime}\right)\right)=(c(I), y(I))$. Therefore

$$
\begin{aligned}
\exp \left(\int_{n^{0}}^{n} \frac{u_{n c}}{u_{c}} d n^{\prime}\right) & =\exp \left(\int_{n^{0}}^{n_{0}(I)} \frac{u_{n c}}{u_{c}} d n^{\prime}+\int_{n_{0}(I)}^{n} \frac{d \ln u_{c}}{d n^{\prime}} d n^{\prime}\right) \\
& =\exp \left(\int_{n^{0}}^{n_{0}(I)} \frac{u_{n c}}{u_{c}} d n^{\prime}+\ln \frac{u_{c}(c(I), y(I), n)}{u_{c}\left(c(I), y(I), n_{0}(I)\right)}\right) \\
& =\frac{u_{c}(c(I), y(I), n)}{u_{c}\left(c(I), y(I), n_{0}(I)\right)} \exp \left(\int_{n^{0}}^{n_{0}(I)} \frac{u_{n c}}{u_{c}} d n^{\prime}\right)
\end{aligned}
$$

For any $I \in \mathcal{I}(n)$, it follows that

$$
\begin{aligned}
& \int_{n_{0}(I)}^{n_{1}(I)} \frac{\psi^{\prime}(n)}{u_{c}(c(n), y(n), n)} e^{\int_{n^{0}}^{n} \frac{u_{n c}}{u_{c}} d n^{\prime}} d n \\
= & \frac{1}{u_{c}\left(c(I), y(I), n_{0}(I)\right)} e^{\int_{n^{0}}^{n_{0}(I)} \frac{u_{n c}}{u_{c}} d n^{\prime}} \int_{n_{0}(I)}^{n_{1}(I)} \psi^{\prime}(n) d n \\
= & \frac{1}{u_{c}\left(c(I), y(I), n_{0}(I)\right)} e^{\int_{n^{0}}^{n_{0}(I)} \frac{u_{n c}}{u_{c}} d n^{\prime}}\left[\psi\left(n_{1}(I)\right)-\psi\left(n_{0}(I)\right)\right] .
\end{aligned}
$$

If $\psi(\bar{n})=0$, then, by the definition of $n_{0}(I)$ and $n_{1}(I)$ and the continuity of $\psi$, one has $\psi\left(n_{0}(I)\right)=\psi\left(n_{1}(I)\right)=0$, hence

$$
\int_{n_{0}(I)}^{n_{1}(I)} \frac{\psi^{\prime}(n)}{u_{c}} e^{\int_{n^{0}}^{n} \frac{u_{n c}}{u_{c}} d n^{\prime}} d n=0
$$


for all $I \in \mathcal{I}$. Given that the set $\mathcal{I}$ is at most countable, (A.18) and (A.20) imply

$$
\int_{\bar{n}}^{\hat{n}} \frac{\psi^{\prime}(n)}{u_{c}} f(n) e^{\int_{n}^{n} \frac{u_{n c}}{u_{c}} d n^{\prime}} d n=0 .
$$

If $\psi(\bar{n})<0$, there exists an initial interval $I_{1}$ of constancy of $c(\cdot)$ such that $\bar{n}=n_{0}\left(I_{1}\right)$. For $I \in \mathcal{I} \backslash\left\{I_{1}\right\}$, one again has $\psi\left(n_{0}(I)\right)=\psi\left(n_{1}(I)\right)=0$, so (A.20) holds and (A.18) - (A.20) imply

$$
\begin{aligned}
\int_{\bar{n}}^{\hat{n}} \frac{\psi^{\prime}(n)}{u_{c}} f(n) e^{\int_{n^{0}}^{n} \frac{u_{n c}}{u_{c}} d n^{\prime}} d n & =\int_{\bar{n}}^{n_{1}\left(I_{1}\right)} \frac{\psi^{\prime}(n)}{u_{c}} f(n) e^{\int_{n^{0}}^{n} \frac{u_{n c}}{u_{c}} d n^{\prime}} d n \\
& =\frac{1}{u_{c}(c(I), y(I), \bar{n})} e^{\int_{n^{0}}^{\bar{n}} \frac{u_{n c}}{u_{c}} d n^{\prime}}\left[\psi\left(n_{1}\left(I_{1}\right)\right)-\psi(\bar{n})\right] .
\end{aligned}
$$

By the definition of $n_{1}\left(I_{1}\right)$ and the continuity of $\psi$, one has $\psi\left(n_{1}\left(I_{1}\right)\right)=0$. Therefore $\psi(\bar{n})<0$ implies

$$
\int_{\bar{n}}^{\hat{n}} \frac{\psi^{\prime}(n)}{u_{c}} f(n) e^{\int_{n}^{n} \frac{u_{n c}}{u_{c}} d n^{\prime}} d n>0 .
$$

Proof of Lemma 5.3. If the lemma is false, $\lambda=0$. Then (5.15) can be written as

$$
\frac{\psi^{\prime}(n)}{u_{c}}+f(n)+\varphi^{\prime}(n)+\varphi(n) \frac{u_{n c}}{u_{c}} \leq 0,
$$

which in turn implies

$$
\frac{\psi^{\prime}(n)}{u_{c}} e^{\int_{n_{0}}^{n} \frac{u_{n c}}{u_{c}} d n^{\prime}}+\frac{d}{d n}\left(\varphi(n) e^{\int_{n_{0}}^{n} \frac{u_{n c}}{u_{c}} d n^{\prime}}\right)<0 .
$$

By integration, one obtains

$$
\int_{n^{0}}^{n^{1}} \frac{\psi^{\prime}(n)}{u_{c}} e^{\int_{n^{0}}^{n} \frac{u_{n c}}{u_{c}} d n^{\prime}} d n+\varphi\left(n_{1}\right) e^{\int_{n^{0}}^{n^{1}} \frac{u_{n c}}{u_{c}} d n^{\prime}}-\varphi\left(n^{0}\right)<0 .
$$

By (5.12), therefore,

$$
\int_{n^{0}}^{n^{1}} \frac{\psi^{\prime}(n)}{u_{c}} e^{\int_{n^{0}}^{n} \frac{u_{n c}}{u_{c}} d n^{\prime}} d n<0
$$

However, by Lemma A.4, in combination with (5.13) and (5.9), one also has

$$
\int_{n^{0}}^{n^{1}} \frac{\psi^{\prime}(n)}{u_{c}} e^{\int_{n^{0}}^{n} \frac{u_{n c}}{u_{c}} d n^{\prime}} d n \geq 0 .
$$

The assumption that $\lambda=0$ has thus led to a contradiction and must be false. 


\section{References}

[1] Besley, T., and S. Coate (1995), The Design of Income Maintenance Programmes, Review of Economic Studies 62, 187 - 221.

[2] Brunner, J.K. (1993), A Note on the Optimal Income Tax, Journal of Public Economics 50, 445 - 451.

[3] Brunner, J.K. (1995), A Theorem on Utilitarian Redistribution, Social Choice and Welfare 12, 175 - 179.

[4] Christiansen, V. (1983), Some Important Properties of the Social Marginal Utility of Income, Scandinavian Journal of Economics 85, 359 371.

[5] Ebert, U. (1992), A Reexamination of the Optimal Nonlinear Income Tax, Journal of Public Economics 49, 47 - 73.

[6] Edgeworth, F.Y. (1897/1925), The Pure Theory of Taxation, Economic Journal (1897), reprinted in: Papers Relating the Political Economy, Macmillan, London (1925), 110 - 116.

[7] Guesnerie, R. (1995), A Contribution to the Pure Theory of Taxation, Econometric Society Monograph, Cambridge University Press, Cambridge, U.K.

[8] Guesnerie, R., and J. Seade (1982), Nonlinear Pricing in a Finite Economy, Journal of Public Economics 17, 157 - 179.

[9] Guesnerie, R., and J.-J. Laffont (1984), A Complete Solution to a Class of Principal-Agent Problems with an Application to the Control of a Self-Managed Firm, Journal of Public Economics 25, 329 - 369.

[10] Hammond, P. (1979), Straightforward Individual Incentive Compatibility in Large Economies, Review of Economic Studies 46, 263 - 282.

[11] Hellwig, M.F. (1986), The Optimal Linear Income Tax Revisited, Journal of Public Economics 31, 163 - 179.

[12] Hellwig, M.F. (2005a), A Maximum Principle for Control Problems with Monotonicity Constraints, in preparation, Max Planck Institute for Research on Collective Goods, Bonn, Germany. 
[13] Hellwig, M.F. (2005b), Optimal Income Taxation for Mixed Type Distributions, in preparation, Max Planck Institute for Research on Collective Goods, Bonn, Germany.

[14] Homburg, S. (2004), The Optimal Income Tax: Restatement and Extensions, mimeo, University of Hannover, Hannover, Germany.

[15] Laroque, G. (2005), Income Maintenance and Labor Force Participation, Econometrica 73, 341 - 376.

[16] Matthews, S., and J. Moore (1987), Monopoly Provision of Quality and Warranties: An Exploration in the Theory of Multidimensional Screening, Econometrica 55, 441 - 467.

[17] Milgrom, P.R., and C.M. Shannon (1994), Monotone Comparative Statics, Econometrica 62, 157 - 180.

[18] Mirrlees, J.M. (1971), An Exploration in the Theory of Optimum Income Taxation, Review of Economic Studies 38, 175 - 208.

[19] Mirrlees, J.M. (1976), Optimal Tax Theoy: A Synthesis, Journal of Public Economics 6, 327 - 358.

[20] Roëll, A. (1985), A Note on the Marginal Tax in a Finite Economy, Journal of Public Economics 28, 267 - 272.

[21] Saez, E. (2002), Optimal Income Transfer Programs: Intensive versus Extensive Labor Supply Responses, Quarterly Journal of Economics 117, 1039 - 1073.

[22] Seade, J. (1977), On the Shape of Optimal Tax Schedules, Journal of Public Economics 7, 203 - 236.

[23] Seade, J. (1982), On the Sign of the Optimum Marginal Income Tax, Review of Economic Studies 49, 637 - 643.

[24] Stiglitz, J.E. (1982), Self-Selection and Pareto Efficient Taxation, Journal of Public Economics 17, 213 - 240.

[25] Werning, I. (2000), An Elementary Proof of Positive Optimal Marginal Taxes, mimeo, University of Chicago.

[26] Weymark, J. (1986), A Reduced-Form Optimal Nonlinear Income Tax Problem, Journal of Public Economics 30, 199 - 217. 